\title{
COPRODUCTS AND SOME UNIVERSAL RING CONSTRUCTIONS $\left({ }^{1}\right)$
}

BY

\author{
GEORGE M. BERGMAN
}

\begin{abstract}
Let $R$ be an algebra over a field $k$, and $P, Q$ be two nonzero finitely generated projective $R$-modules. By adjoining further generators and relations to $R$, one can obtain an extension $S$ of $R$ having a universal isomorphism of modules, $i: P \otimes_{R} S \cong Q \otimes_{R} S$.

We here study this and several similar constuctions, including (given a single finitely generated projective $R$-module $P$ ) the extension $S$ of $R$ having a universal idempotent module-endomorphism $e: P \otimes S \rightarrow P \otimes S$, and (given a positive integer $n$ ) the $k$-algebra $S$ with a universal $k$-algebra homomorphism
\end{abstract} of $R$ into its $n \times n$ matrix ring, $f: R \rightarrow m_{n}(S)$.

A trick involving matrix rings allows us to reduce the study of each of these constructions to that of a coproduct of rings over a semisimple ring $\boldsymbol{R}_{\mathbf{0}}$ ( $=k \times k \times k, k \times k$, and $k$ respectively in the above cases), and hence to apply the theory of such coproducts. As in that theory, we find that the homological properties of the construction are extremely good: The global dimension of $S$ is the same as that of $R$ unless this is 0 , in which case it can increase to 1 , and the semigroup of isomorphism classes of finitely generated projective modules is changed only in the obvious fashion; e.g., in the first case mentioned, by the adjunction of the relation $[P]=[Q]$.

These results allow one to construct a large number of unusual examples.

We discuss the problem of obtaining similar results for some related constructions: the adjunction to $R$ of a universal inverse to a given homomorphism of finitely generated projective modules, $f: P \rightarrow Q$, and the formation of the factor-ring $R / T_{P}$ by the trace ideal of a given finitely generated projective $R$-module $P$ (in other words, setting $P=0$ ).

The idea for a category-theoretic generalization of the ideas of the paper is also sketched.

Received by the editors October 15, 1973.

AMS (MOS) subject classifications (1970). Primary 16A00, 16A50, 16A54, 16A64, 18F25; Secondary 08A10, 08A25, 16A06, 16A08, 16A32, 16A42, 16A48, 16A60, $18 \mathrm{E} 05$.

Key words and phrases. Universal module homomorphism (isomorphism), finitely generated projective module, matrix ring, coproduct of rings, representable functor, semigroup of $R$-modules (resp. projective $R$-modules) under “ $\oplus$ ", global dimension, $n$-fir, localization, trace ideal of a projective module, $k$-linear category.

(1) The idea on which this paper is based occurred to the author while he was supported by a fellowship from England's Science Research Council (1969-70). Most of the preparation for publication was done while the author was an Alfred P. Sloan Fellow. 
1. Introduction. It is well known that there exist (nonzero) noncommutative rings $R$ such that the free right $R$-modules of two distinct ranks, $R^{m}$ and $R^{n}$, are isomorphic as modules. A module-isomorphism $i: R^{n} \rightarrow R^{m}$ can be represented by an $m \times n$ matrix $\left(\left(x_{i j}\right)\right)$ having an $n \times m$ inverse matrix $\left(\left(y_{i j}\right)\right)$, and W. G. Leavitt observed in [25] that to study this phenomenon one could introduce the ring (or more generally, algebra over a fixed commutative base-ring $k$ ) $V_{m, n}$ defined by $2 m n$ generators $a_{i j}(i \leqslant m ; j \leqslant n), b_{i j}(i \leqslant n ; j \leqslant m)$ and the $m^{2}+$ $n^{2}$. relations saying that the product matrices $\left(\left(a_{i j}\right)\right)\left(\left(b_{i j}\right)\right)$ and $\left(\left(b_{i j}\right)\right)\left(\left(a_{i j}\right)\right)$ are the $m \times m$ and $n \times n$ identity matrices. That is:

$$
\begin{aligned}
& \sum_{i=1}^{m} a_{h i} b_{i j}=\delta_{i j} \quad(h, j=1, \cdots, m), \\
& \sum_{i=1}^{n} b_{h i} a_{i j}=\delta_{i j} \quad(h, j=1, \cdots, n) .
\end{aligned}
$$

These $k$-algebras $V_{m, n}$ (for $k$ a field), and the related algebras $U_{m, n}$, with a universal left-invertible map from the free module of rank $n$ to that of rank $m$, and $W_{m}$ with a universal idempotent $m \times m$ matrix, have been further studied by P. M. Cohn [13], [15] and L. A. Skornyakov [30]. The results of this paper include and strongly generalize those of these earlier investigations. Given a $k$ algebra $R$, we shall study the rings obtained by "adjoining to $R$ " universal homomorphisms, isomorphisms, left-invertible maps, and idempotent endomorphisms between finitely generated projective $R$-modules, and further related constructions.

The idea of our approach will be to use the Morita equivalence between a $k$-algebra $R$ and its $s \times s$ matrix ring $\mathrm{m}_{s}(R)$ for appropriate $s$, to take us from the general case of our problem to the special case where the projective right modules under study are direct summands in the free right $R$-module of rank 1 . Thus, if we are studying the adjunction of an isomorphism between projective modules $P$ and $Q$, we reduce to the case where $R$ has orthogonal idempotents $e_{P}$ and $e_{Q}$ such that $P=e_{P} R, Q=e_{Q} R$.

Then $R=e_{P} R \oplus e_{Q} R \oplus e_{U} R\left(e_{U}=1-e_{P}-e_{Q}\right.$; direct sum as right ideals) and the map $(a, \beta, \gamma) \mapsto a e_{P}+\beta e_{Q}+\gamma e_{U}$ is a ring homomorphism $k \times k \times k \rightarrow R$; and in terms of this map, the direct summands $e_{P} R$ and $e_{Q} R$ of $R$ are induced by the summands $k \times 0 \times 0$ and $0 \times k \times 0$ of $k \times k \times k$. Now the universal extension of the $k$-algebra $k \times k \times k$ by an isomorphism of the module $k \times 0 \times 0$ with $0 \times k \times 0$ is easily shown to be the ring $\mathrm{m}_{2}(k) \times k$, where $e_{P}$ and $e_{Q}$ are identified with the matrix units $e_{11}$ and $e_{22}$ of $\mathrm{m}_{2}(k)$, and the isomorphisms introduced are $e_{21}$ and $e_{12}$. The desired universal extension of $R$ 
can now be obtained as the coproduct, over $k \times k \times k$, of $R$ with this extension $\mathrm{m}_{2}(k) \times k$. The results of [7] give us plentiful information about the module theory of this coproduct.

A word of caution: It is misleading to speak of, say, the ring $V_{m, n}$ mentioned above as the universal $k$-algebra $R$ such that $R^{m} \cong R^{n}$ as modules. Rather, it is the k-algebra $R$ with a universal isomorphism $i: R^{m} \rightarrow R^{n}$. The first statement would imply that for every $k$-algebra $S$ such that $S^{m} \cong S^{n}$ there should exist a unique homomorphism $f: R \rightarrow S$. Rather, for each such $S$ and each isomorphism $j: S^{m} \rightarrow S^{n}$ we get a unique homomorphism $f: R \rightarrow S$ such that $j=i \otimes_{f} S$. A $k$-algebra with the former property does not exist. (Even above, I was imprecise at one point: I said $U_{m, n}$ had a universal left-invertible map $a: R^{n} \rightarrow R^{m}$. What I should have said is that it has a universal pair of maps, $a: R^{n} \rightarrow R^{m}, b: R^{m} \rightarrow R^{n}$ such that $b a=1_{R^{n}}$. The point is that left inverses are not unique, and to determine a $k$-algebra homomorphism $R \rightarrow$ $S$, one must specify not only the module-map $S^{n} \rightarrow S^{m}$ to which a goes, but also the left inverse of $a$ to which $b$ should be carried.)

In $\$ 7$ we consider another type of construction. Given a $k$-algebra $R$ and an integer $n$, we construct a $k$-algebra $S=\mathfrak{w}_{n}(R)$ with a universal map $R \rightarrow$ $\mathrm{m}_{n}(S)$, that is, a "universal coefficient ring for $n \times n$ matrix representations of $R$." Category-theoretically, $\mathfrak{w}_{n}$ is the left adjoint of the $n \times n$ matrix-ring functor $\mathrm{m}_{n}: k$-algebras $\rightarrow k$-algebras. Again, $\mathfrak{w}_{n}$ can be reduced to a combination of coproducts and matrix-ring functors. We find that not only does this functor preserve many module-theoretic.properties, but $\mathfrak{w}_{n}(R)$ is always an $(n-1)$-fir, in particular, if $n \geqslant 2$, an integral domain. It follows that most sorts of homological properties, pathological or otherwise, that can be exhibited by a $k$-algebra can be exhibited by one that is an integral domain ( $\S 8)$. E.g., Small [31] has constructed a right hereditary non-left-semihereditary $k$-algebra $R$; $\mathfrak{m}_{2}(R)$ will have these same properties and also have no zero-divisors.

In $\S 9$ we apply a theorem of Cohn's to show that a large class of rings of the sorts discussed above satisfy $n$-term weak algorithm.

$\S \S 10$ and 11 discuss the possibility of obtaining results similar to some of the present ones for the constructions of adjoining a (right, left or 2-sided) inverse to a given map of projective modules, and of putting a projective module equal to zero (= dividing out by its trace ideal).

In $\S 12$ we show how the idea that a $k$-linear category is a generalized $k$ algebra (cf. [28]) can provide a unifying framework for all the results of this paper and of [7] and can suggest some strong generalizations thereof.

In an appendix, $\S 13$, the special role of finitely generated projective modules in these universal constructions is examined. 
2. Some terminology and conventions. All rings will be associative with 1 , and all modules unital. When the contrary is not mentioned, "module" will mean "right module". Homomorphisms of rings or modules will be written on the left, and composed accordingly.

Recall that for $k$ a commutative ring a $k$-algebra can be described as a ring $R$ given with a homomorphism of $k$ into its center, while for $R$ an arbitrary ring, a ring $S$ given with a homomorphism $R \rightarrow S$ is called an $R$ ring. We shall need a term for a combination of these two concepts. If $R$ is a $k$-algebra, then a $k$-algebra $S$ given with a $k$-algebra homomorphism $R \rightarrow S$ will be called an $R$-ring ${ }_{k}$. (In effect, "ring ${ }_{k}$ " is being used as an abbreviation for $k$-algebra where the latter expression would be clumsy.)

If $S$ is an $R$-ring and $M$ an $R$-module, then the $S$-module $M \otimes_{R} S$ will often be abbreviated $\bar{M}$ if there is no danger of confusion. Likewise, if $f: M$ $\rightarrow N$ is a homomorphism of $R$-modules, then $f \otimes S$ may be abbreviated $\bar{f}$ : $\bar{M} \rightarrow \bar{N}$, and if $x$ is an element of $M$ (or $R$ ) its image in $\bar{M}$ (resp. $S$ ) may be denoted $\bar{x}$. (At times we may simply write $x$; e.g. if for some set $X$ we take $M$ to be the free module on $X, \bigoplus_{X} x R$, then we may write the free $S$-module $\bar{M}$ as $\bigoplus_{X} x S$.)

For any ring $R$, the category of finitely generated right $R$-modules will be written $\operatorname{Mod} R$, as in [7], and the full subcategory of finitely generated projective right $R$-modules will be denoted P-Mod $R$. Further notation will be introduced as needed.

We shall occasionally refer to the concepts of firs ( $=$ free ideal rings), $n$ firs, and $n$-term weak algorithm, but except in $\S 9$, and to an extent $\S \S 7,8$, these concepts will not be essential to an understanding of the main points discussed. For background on these classes of rings, see [16].

3. Universal module-map constructions-general nonsense. Let $k$ be a fixed commutative ring. All the constructions we shall study can be built up from the two described below:

THEOREM 3.1 (AdJOINING MAPS). Let $R$ be a k-algebra, $M$ any $R$ module, and $P$ a finitely generated projective $R$-module. Then there exists an $R$-ring $k_{k}, S$, having a universal module homomorphism $f: M \otimes S \rightarrow P \otimes S$; that is, having an S-module homomorphism $f$ such that given any $R$-ring ${ }_{k}, T$, and any T-module homomorphism $g: M \otimes T \rightarrow P \otimes T$, there exists a unique homomorphism $S \rightarrow T$ of $R$-rings ${ }_{k}$ such that $g=f \otimes_{S} T$.

More generally, given a family of such pairs of modules, $M_{i}, P_{i}(i \in I, a$ set) there exists an $R$-ring ${ }_{k} S$ having a universal family of homomorphisms $\left\{f_{i}: M_{i} \otimes S \rightarrow P_{i} \otimes S \mid i \in I\right\}$ with the same universal property. 
TheOREM 3.2 (IMPOSING RELATIONS). Let $R$ be a k-algebra, $M$ any $R$ module, $P$ a projective $R$-module, and $f: M \rightarrow P$ any module homomorphism. Then there exists an $R$-ring ${ }_{k} S$ such that $f \otimes S=0$, and universal for that property: Given any $R$-ring $T$ with $f \otimes T=0$, there exists a unique homomorphism of $R$-rings, $S \rightarrow T$.

More generally, given a family of such $R$-module maps, $f_{i}: M_{i} \rightarrow P_{i}(i \in$ $I$, any class), there exists an $R$-ring $k_{k}$ universal for the property: For all $i \in I$, $f_{i} \otimes_{R} S=0$ (in fact, universal for this property as an $R \cdot$ ring).

Proofs. In the situation of Theorem 3.1, let us write $M$ as the cokernel of a map $u$ of free $R$-modules, say on bases $A$ and $B: \bigoplus_{A} a R \stackrel{u}{\rightarrow} \bigoplus_{B} b R$ $\rightarrow M$, and for each $a \in A$, let $u(a)=\Sigma_{B} b u_{b a}$ ( $u_{b a} \in R$, zero for almost all $b$ ). Then for any $R$-ring $T, M \otimes T$ has the presentation $\bigoplus_{A} a T \stackrel{\bar{u}}{\rightarrow}$ $\bigoplus_{B} b T \rightarrow \bar{M}$.

Let us write the finitely generated projective module $P$ as a direct summand in a free $R$-module of finite rank $\bigoplus_{C} c R=P \oplus Q$; specifically, as the kernel of an idempotent endomorphism $e$ defined by $e(c)=\Sigma_{c^{\prime} \in C} c^{\prime} e_{c^{\prime} c}$ $\left(e_{c^{\prime} c} \in R\right)$. Then for any $R$-ring $T$, the map $\bar{e}$ with coefficients $\bar{e}_{c^{\prime} c}$ will induce the decomposition $\bigoplus_{C} c T=P \otimes T \oplus Q \otimes T$.

Hence for any $R$-ring $T$, a module homomorphism $g: M \otimes T \rightarrow P \otimes T$ will be uniquely determined by a map $h: \bigoplus_{B} b T \rightarrow \bigoplus_{C} c T$ which left annihilates $\bar{u}$ and right annihilates $\bar{e}$; equivalently (since $C$ is finite) by any family of elements $h_{c b} \in T$ satisfying

$$
\sum_{B} h_{c b} u_{b a}=0 \quad(a \in A, c \in C) \quad \text { and } \quad \sum_{c \in C} e_{c^{\prime} c^{\prime} h_{c b}}=0\left(c^{\prime} \in C, b \in B\right) \text {. }
$$

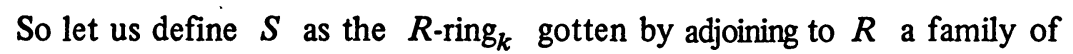
generators $h_{c b}(c \in C, b \in B)$ subject to the above relations. Then these elements will constitute the coordinates of an $S$-module homomorphism $f: M \otimes S$ $\rightarrow P \otimes S$ with the desired universal property. The case of an arbitrary indexed family of maps is clearly no more difficult.

In the case of Theorem 3.2, we again write $P$ as a direct summand in a free module $\bigoplus_{C} c R$. The fact that it is a direct summand insures that for any $R$-ring $T$, the map $i: P \otimes T \rightarrow \bigoplus_{C} c T$ induced by the inclusion $i: P \rightarrow$ $\bigoplus_{C} c R$ is again 1-1. Hence if we write for each $m \in M, f(m)=\Sigma c f_{c m}$ $\left(f_{c m} \in R\right)$, we can see that for any $R$-ring $T, f \otimes T$ will be zero if and only if all the $f_{c m}$ go to zero in $T$. So the desired universal ring $S$ is simply the quotient of $R$ by the two-sided ideal generated by these elements. Again, the case of a family of maps is no different, (even if this family is indexed by a proper class-since the coefficients $f_{c m}$, belonging to $R$, will form a set). 
(In $\S 13$ we shall show why $P$ must be assumed finitely generated and projective in Theorem 3.1, and make some related observations on Theorem 3.2.)

Note that when $M$ and $P$ are both $R$, the free module of rank 1, then the construction of Theorem 3.1 is that of adjoining an indeterminate, and the construction of Theorem 3.2 is that of imposing a relation, in the usual senses.

From the point of view of the categorist, Theorem 3.1 says that the functor from $R$-rings ${ }_{k}$ to sets, associating to $T$ the set $\operatorname{Hom}_{T}(M \otimes T, P \otimes T)$, is representable.

The ring $S$ constructed in Theorem 3.1 will be denoted $R\langle f: M \otimes \rightarrow$ $P \otimes\rangle$ or $R\langle f: \bar{M} \rightarrow \bar{P}\rangle$, and that of Theorem 3.2, $R\langle f \otimes=0\rangle$ or $R\langle\bar{f}=0\rangle$. These are well defined up to natural isomorphism. Note that if $\alpha: M \cong M^{\prime}$, $\beta: P \cong P^{\prime}$ are isomorphisms of $R$-modules, the rings $R\langle f: \bar{M} \rightarrow \bar{P}\rangle$ and $R\left\langle f^{\prime}: \bar{M}^{\prime} \rightarrow \bar{P}^{\prime}\right\rangle$ are isomorphic under the unique map of $R$-rings taking the module-map $f$ to $\bar{\beta}^{-1} f^{\prime} \bar{\alpha}$. The analogous observation holds for the second construction.

Now that we can introduce generators and relations, we can describe more complicated constructions. Given a $k$-algebra $R$ and a homomorphism $f$ : $P \rightarrow Q$ between two finitely generated projective $R$-modules, we may construct an $R$-ring - $_{k} R\left\langle(f \otimes)^{-1}\right\rangle$ or $R\left\langle\bar{f}^{-1}\right\rangle$ in which $\bar{f}$ becomes an isomorphism, by adjoining a map $g: \bar{Q} \rightarrow \bar{P}$, then setting $1_{\bar{P}}-g \bar{f}$ and $1_{\bar{Q}}-\bar{f} g$ equal to zero. If one only sets the first of these equal to zero, one gets a ring $R\left\langle g ; g \bar{f}=1_{\bar{P}}\right\rangle$ with a universal left inverse to $\bar{f}$. There $\bar{f}$ becomes an isomorphism of $\bar{P}$ and a direct summand of $\bar{Q}$, with a distinguished cosummand $\operatorname{Ker} \bar{f} g$, in a universal manner. Imposing instead the other equality gives $R\left\langle g ; \bar{f} g=1_{\bar{Q}}\right\rangle$, were $g \bar{f}$ gives the projection of $\bar{P}$ onto a direct summand, which is isomorphic to $\bar{Q}$ under $\bar{f}$, and is given with a distinguished cosummand $\operatorname{Ker} g \bar{f}$. If $f$ was an isomorphism to begin with all three of these universal constructions leave $R$ unchanged; but if $f$ was (only) one-sided invertible, it is not hard to show that all three will change $R$.

Given a $k$-algebra $R$ and two finitely generated projective $R$-modules $P$ and $Q$, we can adjoin a universal isomorphism between $P \otimes$ and $Q \otimes$ by first freely adjoining a map $P \otimes \rightarrow Q \otimes$, then adjoining an inverse. We shall call the resulting $R$-ring ${ }_{k} R\left\langle i, i^{-1}: \bar{P} \cong \bar{Q}\right\rangle$. Here if $P$ and $Q$ were already isomorphic $R$-modules, the construction is equivalent to universally adjoining an automorphism of $P$. E.g., if $P$ and $Q$ are both the free right $R$-module of rank 1 , the new $R$-ring ${ }_{k}$ is the $k$-algebra $R\left\langle x, x^{-1}\right\rangle$ obtained by freely adjoining to $R$ one indeterminate and an inverse thereto. Again, one can adjoin a map $\bar{P} \rightarrow \bar{Q}$ and a one-sided inverse only, getting a ring $R\langle i: \bar{P} \rightarrow \bar{Q}, j: \bar{Q} \rightarrow \bar{P} ; j i=1 \bar{P}$.

Given a single finitely generated projective module $P$, one may obtain an 
$R$-ring ${ }_{k}$ with a universal direct sum decomposition $\bar{P}=P_{1} \oplus P_{2}$ by adjoining to $R$ a universal idempotent endomorphism of $P \otimes$. This ring will be written $R\left\langle e: \bar{P} \rightarrow \bar{P} ; e^{2}=e\right\rangle$. Or one can annihilate $P$ entirely by setting its identitymap equal to zero. The resulting ring, $R\left\langle 1_{\bar{P}}=0\right\rangle$ or simply $R\langle\bar{P}=0\rangle$ is easily seen, from its universal property or by examination of the construction, to be the quotient $R / T_{P}$ of $R$ by the trace ideal of $P$.

Of the above constructions, we shall obtain "good" module-theories in $\S 5$ for those of adjoining a universal map, $R\langle f: \bar{P} \rightarrow \bar{Q}\rangle$, adjoining a universal isomorphism, $R\left\langle i, i^{-1}: \bar{P} \cong \bar{Q}\right.$ ) (when $P$ and $Q$ are nonzero finitely generated projective modules) adjoining one-sided invertible maps, $R(i: \bar{P} \rightarrow \bar{Q}, j: \bar{Q} \rightarrow$ $\left.\bar{P} ; j i=1_{\vec{P}}\right\rangle$ (when $Q \neq 0$ ) and adjoining a universal direct-sum decomposition, $R\left\langle e: \bar{P} \stackrel{\vec{P}}{\rightarrow} \bar{P} ; e^{2}=e\right\rangle$. The base-ring $k$ is assumed a field in each case.

On the other hand we cannot expect the operation of adding an arbitrary relation $\bar{f}=0$ to a ring to preserve good homological properties, since one can get arbitrary $k$-algebras by imposing relations on free associative algebras.

Concerning the operations of adjoining a one- or two-sided inverse to a given map $f$, and of killing (the identity map of) a projective module, I shall say what little I know in $\S \S 10,11$.

Let us put all the above constructions into a general framework. Define a $k$-linear category (for $k$ an arbitrary commutative ring) as a category in which every $\operatorname{Hom}$-set $\operatorname{Hom}(X, Y)$ is given a structure of $k$-module, such that the composition maps, $\operatorname{Hom}(Y, Z) \times \operatorname{Hom}(X, Y) \rightarrow \operatorname{Hom}(X, Z)$ are $k$-bilinear. A $k$-linear functor will mean a functor between $k$-linear categories that maps Homsets by $k$-module homomorphisms. In particular, for $R$ a $k$-algebra the category of all $R$-modules has a natural $k$-linear structure, hence so do the full subcategories $\operatorname{Mod} R$ of finitely generated $R$-modules, and P-Mod $R$ of finitely generated projective $R$-modules. If $T$ is an $R$-ring ${ }_{k}$, the restriction of scalars functor from $T$-modules to $R$-modules, and the functor $\_\otimes T$ going the other way, are clearly $k$-linear.

The construction of the following theorem may be obtained by one application of Theorem 3.1 to obtain all the morphisms needed, followed by one application of Theorem 3.2 for relations.

The reader should be able to express the constructions discussed above as examples of this construction!

THEOREM 3.3. Let $R$ be a k-algebra, and let us be given small k-linear categories $A$ and $B$, and k-linear functors $F: A \rightarrow P$-Mod $R$ and $G: A$ $\rightarrow B$, such that $G$ is a bijection on objects. Then there exist an R-ring $k_{k}$, $S=R\langle B\rangle$ and a k-linear functor $H: B \rightarrow$ P-Mod $S$ which make the following diagram commute: 


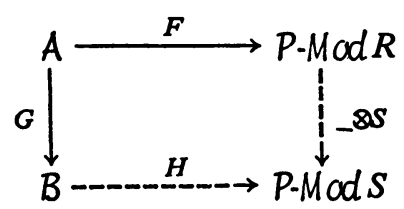

and which are universal for this property.

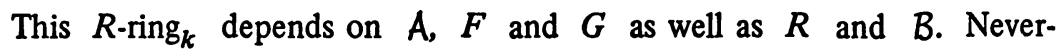
theless we shall use the compact symbol $R\langle B\rangle$; there will be little occasion for confusion, and the notation is suggestive since it is the morphisms and relations of $B$ that are being "adjoined" to $R$. (For specific cases of this construction we shall continue to write $R\left\langle i, i^{-1}: \bar{P} \cong \bar{Q}\right\rangle$ etc.)

Note that if $F, F^{\prime}: A \rightarrow P-M o d R$ are isomorphic functors, they yield isomorphic extension rings. (Cf. earlier discussion of " $\alpha: P \cong P^{\prime}$.")

By comparison of universal properties, one verifies without difficulty the behavior of the above construction with respect to change of $R$. This is where coproducts come in:

THEOREM 3.4. Let $R, A, B, F, G$ be as above, and $R^{\prime}$ any $R$-ring $k^{\text {. Then }}$ $R^{\prime}\langle B\rangle$ may be identified with the coproduct $R^{\prime} \Perp_{R} R\langle B\rangle$ of $R^{\prime}$ with $R\langle B\rangle$ over $R$ :

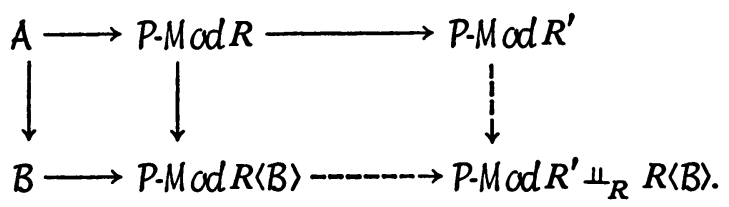

Finally, note that any universal construction for finitely generated projective right $R$-modules is equivalent to a dual construction for finitely generated projective left $R$-modules, because of the well-known contravariant equivalence (duality) between the categories of such modules, given by "“*” = Hom $(,, R)$. (Here it is understood that all homomorphisms of modules are written on the left and composed accordingly. An alternative, and frequently convenient convention would be to write homomorphisms of left modules on the right and so compose them. Then "*" becomes covariant, but we have to work with categories with two different conventions on composition, or else set up unnatural definitions of domain and codomain in the category of left modules! So we shall stick to left-hand notation here.) Introducing superscripts ${ }^{(}()$and ${ }^{(l)}$ for the moment, to distinguish right and left module constructions, we have:

THEOREM 3.5. Under the hypotheses of Theorem 3.3, the universal rings $S=R\langle B\rangle^{(r)}$ and $S^{\prime}=R\left\langle B^{o p}\right\rangle^{(l)}$ associated with the following diagrams: 

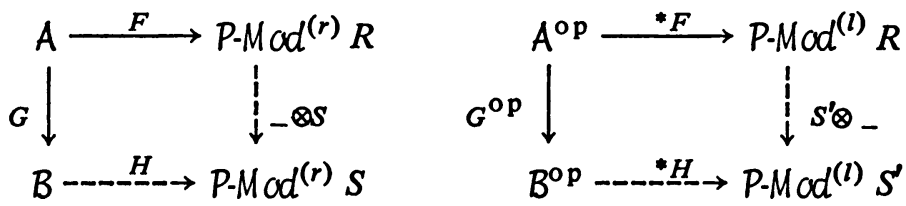

are naturally isomorphic.

Between logical right-left symmetry and the above duality by "**", we find that the study of the properties of right or left modules over a ring obtained by a universal construction on right or left projective modules can be reduced to a case of right modules and a right-universal construction. Namely, first reduce, by logical symmetry, to the case where the question is one of right module theory. Then if after this reduction the construction involves projective left modules, use the above theorem to translate it into a construction on projective right modules. E.g., left inversion of a map of left modules = right inversion of a map of right modules. Many of the constructions we shall study below are self dual in this sense: Making the finitely generated right module $P$ a direct summand in, or isomorphic to the module $Q$ is equivalent to making the left module $P^{*}$ a direct summand in, or isomorphic to the left module $Q^{*}$. Hence these constructions have the same homological behavior on the right and left.

So we shall say little more about left modules, leaving the obvious observations to the reader who has need of them.

4. Matrix rings. For $n$ a positive integer, $m_{n}: \operatorname{Rin} g_{k} \rightarrow R i n g_{k}$ will denote the $n \times n$ matrix-ring functor on the category of $k$-algebras.

By functoriality, it is clear that if $R$ is a $k$-algebra, we have on the $k$ algebra $m_{n}(R)$ a natural structure of $m_{n}(k)$-ring $k_{k}$. If we call the algebra together with this additional structure $\mathfrak{M l}_{n}(R)$, we have a factorization of $\mathrm{m}_{n}$ :

$$
\operatorname{Ring}_{k} \stackrel{\mathbb{P}_{n}}{\longrightarrow} m_{n}(k)-\operatorname{Ring}_{k} \stackrel{3_{n}}{\longrightarrow} \operatorname{Ring}_{k},
$$

where $\Im_{n}$ is the forgetful functor.

Recall that the functor $\mathcal{M}\}_{n}$ is in fact an equivalence of categories $[7, \S 10]$. Its inverse (strictly: inverse up to isomorphism) $\mathfrak{M}_{n}^{-1}: \mathfrak{m}_{n}(k)-R$ ing $_{k} \rightarrow$ $\operatorname{Ring}_{k}$ may be constructed as taking an $\mathrm{m}_{n}(k)$-ring ${ }_{k}, R$, to the $k$-algebra $e_{11} R e_{11}$ with unit $e_{11}$ (= the image in $R$ of the matrix unit $e_{11} \in \mathrm{m}_{n}(k)$ ). This is the construction from which the above claim is easiest to verify $[7, \S 10]$. Once one knows that $\mathfrak{g} \mathbb{N}_{n}$ is an equivalence, one sees that its inverse can be constructed more elegantly as the centralizer of $m_{n}(k)$ in $R$.

Further, for $R$ any $k$-algebra, $\operatorname{Mod} R$ and $\operatorname{Mod} m_{n}(R)$ are equivalent $k$-linear categories. (That is, $R$ and $m_{n}(R)$ are Morita equivalent.) Given an 
$R$-module $M$, we define $\mathfrak{r}_{n}(M)$ to be the module of row vectors of length $n$ in the elements of $M$, on which elements of $\mathrm{m}_{n}(R)$ act via the usual rules for multiplying a vector by a matrix, applied to the $R$-module structure of $M$. We may construct $\mathfrak{r}_{n}^{-1}$ by taking a right $\mathrm{m}_{n}(R)$-module $N$ to the $R$-module $N e_{11}$ $[7, \S 10]$. Because $\mathfrak{r}_{n}$ is an equivalence of categories, and in fact extends to the categories of arbitrary (not necessarily finitely generated) $R$ - and $\mathrm{m}_{n}(R)$-modules, it respects projectivity, homological dimension, etc.

Note that $\mathfrak{r}_{n}$ takes the free right $R$-module of rank $n$ to the free right $\mathfrak{m}_{n}(R)$-module of rank 1 . This fact will allow us to make the crucial reduction of finitely generated projective modules to cyclic projective modules in the next section.

The functor $\mathfrak{r}_{n}$ is also natural in $R$, in the sense that, for any homomorphism $R \rightarrow S$ of $k$-algebras, the following diagrams will commute (where r.o.s. means "restriction of scalars"):
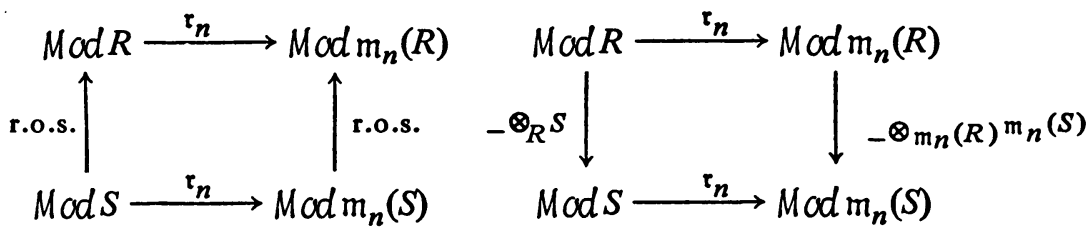

We deduce again by comparing universal properties:

THEOREM 4.1. As in Theorem 3.3, let $R$ be a k-algebra, $A$ and $B$ small (k-linear) categories, and $F: A \rightarrow P$-Mod $R, G: A \rightarrow B$ (k-linear) functors, with $G$ bijective on objects. Let $n$ be a positive integer, and let us map $A$ into P-Mod $\mathrm{m}_{n}(R)$ by $\mathfrak{r}_{n} \circ F$.

Then the resulting universal extension ring, $m_{n}(R)\langle B\rangle$, universal for the second diagram below, may be identified with $\mathrm{m}_{n}(R\langle B\rangle)$, where $R\langle B\rangle$ is constructed from the first diagram:
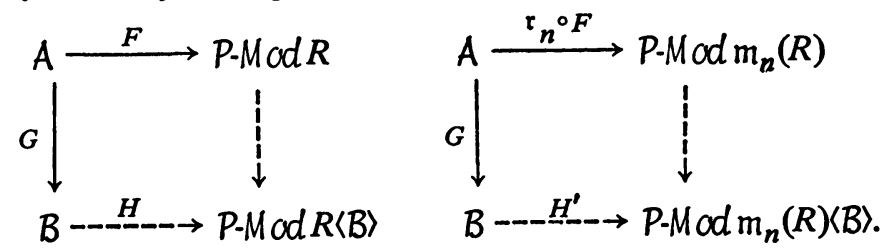

Or, to put the conclusion in the form in which we shall use it in the next section, $R\langle B\rangle$ may be constructed as $\mathfrak{M} l_{n}^{-1}\left(\mathcal{M} l_{n}(R)\langle B\rangle\right)$.

(Strictly, we should only speak of $\mathfrak{m}_{n}(R)\langle B\rangle$, not $\mathcal{M}_{n}(R)\langle B\rangle$, since Theorem 3.1 applies to the category $R \operatorname{in} g_{k}$. But the point here is that we remember the structure of $\mathfrak{m}_{n}(k)$-ring on $\mathfrak{m}_{n}(R)$, and since $\mathfrak{m}_{n}(R)\langle B\rangle$ is constructed as an $\mathfrak{m}_{n}(R)$-ring, it inherits, in particular, a structure of $\mathfrak{m}_{n}(k)$-ring, so we can apply $\mathfrak{M}_{n}^{-1}$ to it. In the theorem, all this is hidden in the phrase "may be identified with"!) 
5. Main theorems. We shall first formulate, then prove Theorems 5.1-5.3. Throughout $\S \S 5-11, k$ will be a field.

First, let $R$ be a $k$-algebra and $P$ a nonzero finitely generated projective right $R$-module. Let $S=R\left\langle e: \bar{P} \rightarrow \bar{P} ; e^{2}=e\right\rangle$. Thus, over $S, \bar{P}=P \otimes S$ decomposes as:

$$
\bar{P}=P_{1} \oplus P_{2} \text { where }\left\{\begin{array}{l}
P_{1}=\operatorname{Im}(e)=\operatorname{Ker}(1-e) \\
P_{2}=\operatorname{Ker}(1-e)=\operatorname{Im}(e) .
\end{array}\right.
$$

We define a fundamental $S$-module to mean an $S$-module obtained as the direct sum of a module $M \otimes_{R} S$ ( $M$ an $R$-module) and of a family (possibly empty, possibly infinite) of copies of $P_{1}$ and $P_{2}$. The full subcategory of all finitely generated $S$-modules which can be given a structure of fundamental module will be denoted Fund $S \subseteq$ Mod $S$. Following the notation of [7], $S_{\oplus}($ Fund $S) \subseteq S_{\oplus}(\operatorname{Mod} S)$ will denote the abelian semigroup of isomorphism classes of finitely generated fundamental $S$-modules under the composition induced by $\oplus$, i.e. $[A]+[B]={ }_{\text {def }}[A \oplus B]$. (Throughout this paper, semigroup will mean semigroup with identity element. When a semigroup is written additively, this element will be denoted 0 .)

THEOREM 5.1. Over the ring $S=R\left\langle e: \bar{P} \rightarrow \bar{P} ; e^{2}=e\right\rangle$, every submodule of a fundamental module is isomorphic to a fundamental module. The abelian semigroup $S_{\oplus}$ (Fund $S$ ) may be obtained from $S_{\oplus}(\operatorname{Mod} R)$ by adjoining two new generators $\left[P_{1}\right]$ and $\left[P_{2}\right]$ and one relation $\left[P_{1}\right]+\left[P_{2}\right]=$ $[P]$. For any $R$-module $M, \mathrm{~h} \operatorname{dim}_{S} \bar{M}=\mathrm{h} \operatorname{dim}_{R} M$; and of course $\mathrm{h} \operatorname{dim}_{S} P_{1}$ $=\mathrm{h} \operatorname{dim}_{S} P_{2}=0$. Globally, $\mathrm{r} \mathrm{gl} \operatorname{dim} S=\mathrm{r} g l \operatorname{dim} R$, unless $\mathrm{r} g l \operatorname{dim} R=0$, in which case $\mathrm{r} g \mathrm{~g} \operatorname{dim} S \leqslant 1$.

Next, let $P$ and $Q$ be two nonzero finitely generated projective modules over a $k$-algebra $R$, and let us form $S=R\left\langle i, i^{-1}: \bar{P} \cong \bar{Q}\right\rangle$. This time, define a fundamental $S$-module to mean one of the form $M \otimes_{R} S$ ( $M$ an $R$-module). This again gives us a full subcategory Fund $S \subseteq \operatorname{Mod} S$, and a subsemigroup $S_{\oplus}($ Fund $S) \subseteq S_{\oplus}(\operatorname{Mod} S)$.

THEOREM 5.2. Over the ring $S=R\left\langle i, i^{-1}: \bar{P} \cong \bar{Q}\right\rangle$, every submodule of a fundamental module is isomorphic to a fundamental module. The abelian semigroup $S_{\oplus}($ Fund $S)$ is obtained from $S_{\oplus}(\operatorname{Mod} R)$ by imposing one relation $[P]=[Q]$. (I.e., the congruence on $S_{\oplus}(\operatorname{Mod} R)$ given by $\{([A],[B]) \mid \bar{A} \cong \bar{B}\}$ is generated by $([P],[Q])$ ). For any $R$-module $M, \mathrm{~h} \operatorname{dim}_{S} \bar{M}=\mathrm{h} \operatorname{dim}_{R} M$. Globally, $\mathrm{r} \mathrm{gl} \operatorname{dim} S=\mathrm{r} \mathrm{gl} \operatorname{dim} R$ unless $\mathrm{r} \mathrm{gl} \operatorname{dim} R=0$, in which case r gl $\operatorname{dim} S \leqslant 1$. 
Finally, again let $P$ and $Q$ be two nonzero finitely generated projective modules over a $k$-algebra $R$, this time take the universal mapping ring $S=R\langle f: \bar{P} \rightarrow \bar{Q}$, and again define a fundamental module to mean one of the form $M \otimes_{R} S$ (M an $R$-module).

THEOREM 5.3. Over the ring $S=R\langle f: \bar{P} \rightarrow \bar{Q}\rangle$, every submodule of a fundamental module is isomorphic to a fundamental module. $S_{\oplus}($ Fund $S) \cong$ $S_{\oplus}(\operatorname{Mod} R)$ under the map $[M] \rightarrow[\bar{M}]$, and this map preserves homological dimensions. $\mathrm{r} \mathrm{gl} \operatorname{dim} S=\max (\mathrm{r} \mathrm{gl} \operatorname{dim} R, 1)$.

Proof of Theorems 5.1-5.3. We first note that for any positive integer $n$, the hypotheses and conclusions of these theorems are respected by the operation $\mathfrak{m}_{n}$ applied to the rings, and $\mathfrak{r}_{n}$ to the modules. Hence by Theorem 4.1, to prove any of these results for a given $R$, it would suffice to prove it for $\mathfrak{m}_{n}(R)$, with $\mathfrak{r}_{n}(P)$ for $P$, etc. Given $R$, let us choose $n$ in the case of Theorem 5.1 to be large enough so that $P$ can be embedded as a proper direct summand in the free right $R$-module of rank $n$, and in the cases of Theorems 5.2 and 5.3, large enough so that $P \oplus Q$ can be so embedded. The $\mathfrak{m}_{n}(R)$ module corresponding to the free $R$-module of rank $n$ is free of rank 1 , hence we are reduced to proving these theorems in the case where $P$, respectively $P \oplus Q$, is a proper direct summand of the free right $R$-module of rank $1, R$. Let us call the complementary summand $U \neq 0$ in each case. We now consider the three theorems one by one.

In the situation to which Theorem 5.1 has been reduced, we have $R=$ $P \oplus U$ as right modules. The projections onto these two summands will be given by left multiplication maps, say by elements $e_{P}, e_{U} \in R$. Thus, $e_{P}, e_{U}$ will be orthogonal idempotents in $R$, with $e_{P}+e_{U}=1$ and $P=e_{P} R, U=$ $e_{U} R$.

The sub-k-algebra of $R$ generated by $e_{P}$ and $e_{U}$ may be identified with $k \times k$, under the map $(\alpha, \beta) \mapsto e_{P} \alpha+e_{U} \beta$. Writing $R_{0}$ for $k \times k$, we note that the $R$-modules $P$ and $U$ are induced by $R_{0}$-modules (in fact, ideals):

$$
\begin{aligned}
& P=e_{P} R \cong P_{0} \otimes_{R_{0}} R, \text { where } P_{0}=k \times 0, \\
& U=e_{U} R \cong U_{0} \otimes_{R_{0}} R, \text { where } U_{0}=0 \times k .
\end{aligned}
$$

It follows, by Theorem 3.4, that $S=R\left\langle e: \bar{P} \rightarrow \bar{P} ; e^{2}=e\right\rangle$ may be constructed as $R \Perp_{R_{0}} R_{0}\left\langle e: \bar{P}_{0} \rightarrow \bar{P}_{0} ; e^{2}=e\right\rangle$. Now the latter factor in this coproduct, the result of adjoining to $k \times k$ a universal idempotent endomorphism of its projective module $k \times 0$, is easily seen to be $(k \times k) \times k$, 
made a $(k \times k)$-ring by the map $(\alpha, \beta) \mapsto(\alpha, \alpha, \beta)$, and with $e$ represented by $(1,0,0) .(2)$ The induced decomposition of the ideal $\bar{P}_{0}=k \times k \times 0$ is as $(1,0,0) k \oplus(0,1,0) k$. The corresponding summands of $\bar{P}$ in $S,(1,0,0) S$ and $(0,1,0) S$, are the desired $P_{1}$ and $P_{2}$. Let us henceforth write $e_{P_{1}}$, $e_{P_{2}}$, and (as before) $e_{U}$ for the elements $e=(1,0,0), e_{P}-e=(0,1,0)$, and $e_{U}=(0,0,1)$ of $k \times k \times k$, and for their images in $S$.

We now turn to $[7, \S 2]$ for information about the module theory of $S=$ $R \Perp_{R_{0}}(k \times k \times k)$. Every module over $k \times k \times k$ is a direct sum of copies of $e_{P_{1}} k, e_{P_{2}} k$ and $e_{U} k$, which induce the $S$-modules $P_{1}, P_{2}$ and $\bar{U}$, hence the "standard modules" of [7] are the $S$-modules of the form $\bar{M} \oplus\left(\oplus P_{1}\right) \oplus$ $\left(\bigoplus P_{2}\right) \oplus(\bigoplus \bar{U})$ ( $M$ an $R$-module. By a symbol like $\bigoplus P_{1}$, we mean a direct sum of copies of $P_{1}$; i.e., $\bigoplus_{I} P_{1}$ for some index-set $I$.) The summand $\bigoplus \bar{U}$ is induced by an $R$-module, hence may be absorbed into the $\bar{M}$; hence the standard modules over our coproduct are, up to isomorphism, what we have here called the fundamental $S$-modules. Our claims about submodules of fundamental modules, and homological and global dimensions, now come right out of [7, Theorem 2.2 and Corollaries $2.4,2.5]$. Corollary 2.8 of that paper tells us that the abelian semigroup $S_{\oplus}($ Fund $S$ ) is given as a pushout by the diagram:

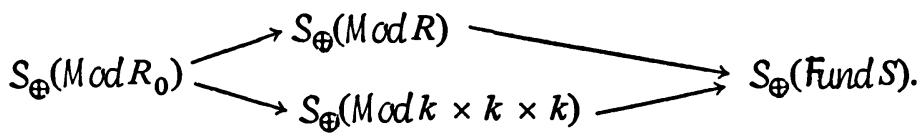

Now $S_{\oplus}\left(\operatorname{Mod} R_{0}\right)$ is the free additive semigroup (with 0$)$ on two generators, $\left[e_{P} k\right]$ and $\left[e_{U} k\right]$. Likewise, $S_{\oplus}(\operatorname{Mod} k \times k \times k)$ is the free abelian semigroup on the three generators $\left[e_{P_{1}} k\right],\left[e_{P_{2}} k\right],\left[e_{U} k\right]$, and can be described as obtained from the former semigroup by adjoining two generators $\left[e_{P_{1}} k\right]$ and $\left[e_{P_{2}} k\right]$, and one relation, $\left[e_{P_{1}} k\right]+\left[e_{P_{2}} k\right]=\left[\overline{e_{P} k}\right]$. Hence the pushout semigroup, $S_{\oplus}($ Fund $S)$, is obtained from $S_{\oplus}(\operatorname{Mod} R)$ by adjoining the images of these two generators, $\left[P_{1}\right]$ and $\left[P_{2}\right]$, and the corresponding relation, $\left[P_{1}\right]+$ $\left[P_{2}\right]=[\bar{P}]$. The proof of Theorem 5.1 is now complete.

The case of $S=R\left\langle i, i^{-1}: \bar{P} \cong \bar{Q}\right\rangle$ given in Theorem 5.2 is quite similar. After our reduction we can write $R=P \oplus Q \oplus U=e_{P} R \oplus e_{Q} R \oplus e_{U} R$, where the $e$ 's are orthogonal idempotents summing to 1 , and this makes $R$ a $(k \times$ $k \times k$ )-ring ${ }_{k}$. The universal extension of $R_{0}=k \times k \times k$ by an isomorphism $i, i^{-1}: e_{P} k \cong e_{Q} k$ is seen to have the form $\mathrm{m}_{2}(k) \times k$, made a $(k \times k \times k)$ -

(2) Note that essentially, this is a consequence of the fact that $k\left\langle e ; e^{2}=e\right\rangle \cong k \times k$. If one wishes to make this argument formal, one should prove a "Theorem 3.6", on direct products of universal-construction rings, saying basically $R_{1}\left\langle B_{1}\right\rangle \times R_{2}\left\langle B_{2}\right\rangle \cong\left(R_{1} \times R_{2}\right) \cdot$ $\left\langle A_{1} \times B_{2}\right\rangle$. The reader will find it easy to do so. I felt that $\S 3$ was getting tedious enough, without putting in a result that we would use in such a trivial way. 
ring via $(\alpha, \beta, \gamma) \mapsto\left(\alpha e_{11}+\beta e_{22}, \gamma\right)$, with $i$ and $i^{-1}$ being given by $\left(e_{21}, 0\right)$ and $\left(e_{12}, 0\right)$. (Again, this is best seen by first dropping the final factor $k$, and verifying that $(k \times k)\left\langle i, i^{-1}: \overline{k \times 0} \cong \overline{0 \times k\rangle}\right.$ is simply $\mathrm{m}_{2}(k)$.) We see that $S_{\oplus}\left(\operatorname{Mod} \mathrm{m}_{2}(k) \times k\right)$ is free on 2 generators, $\left[\mathrm{r}_{2}(k) \times 0\right]$ and $[0 \times k]$, the former being the common image of $\left[e_{P} k\right]$ and $\left[e_{Q} k\right]$, and can be obtained from $S_{\oplus}\left(\operatorname{Mod} R_{0}\right)$ by adjoining the relation $\left[e_{P} k\right]=\left[e_{Q} k\right]$. We see that again the definition of "fundamental module" that we have made characterizes the standard modules in the sense of [7] over the coproduct ring $S$, and our claims follow as before.

The situation for Theorem 5.3, where $S=R\langle f: \bar{P} \rightarrow \bar{Q}\rangle$, is a little different. The corresponding extension of $R_{0}=k \times k \times k$ has the form $\mathrm{t}_{2}(k) \times k$, where $\mathrm{t}_{2}(k)$ denotes the lower triangular matrix ring, $\left(\begin{array}{cc}k & 0 \\ k & k\end{array}\right)$, with $f=$ $\left(e_{21}, 0\right)$. Now $\mathrm{t}_{2}(k)$, and hence $\mathrm{t}_{2}(k) \times k$, has global dimension 1 , that is, it is hereditary, but has nonprojective modules. (In fact, every module over this ring is a direct sum of copies of the obvious projective modules generated by $\left(e_{11}, 0\right),\left(e_{22}, 0\right)$, and $(0,1)$, and of the unique simple nonprojective module, the cokernel of the map $f: \bar{P}_{0} \rightarrow \bar{Q}_{0}$; explicitly, $\left(\left(\begin{array}{ll}0 & 0 \\ k & k\end{array}\right), 0\right) /\left(\left(\begin{array}{ll}0 & 0 \\ 0 & k\end{array}\right), 0\right)$.) Hence the "fundamental" modules over the coproduct ring $S$, as we chose to define them, are not all of the standard modules.

The solution to this difficulty is to use Corollaries 2.10 and 2.11 of [7] in place of Theorem 2.1 and Corollary 2.8. As noted there, the projective modules over any $R_{0}$-ring form an " $R_{0}$-stable class". In the case of $\mathrm{t}_{2}(k) \times k$, we note that the natural map from $S_{\oplus}\left(\operatorname{Mod} R_{0}\right)$ to $S_{\oplus}$ of this class of modules is an isomorphism. It is easy to deduce that the class of modules over the coproduct ring $S$ induced by this class of modules over $\mathrm{t}_{2}(k) \times k$, and all modules over $R$, is precisely the class we have called "fundamental". The indicated corollaries (along with Corollaries 2.4 and 2.5 as before) now give the asserted results. The proof of Theorems 5.1-5.3 is complete.

(Note that the idempotent $e_{U}$, and the corresponding final factor " $-\times k$ " have been inactive throughout these proofs. We needed $U$ merely to enlarge $P$ or $P \oplus Q$ to a free module. We avoided having to distinguish cases by assuming without loss of generality that $U$ was always nonzero. However, we see that when $P$, respectively $P \oplus Q$ is already free, we can carry out our constructions without any " $U$ ", and thus in a slightly simpler, and perhaps pedagogically preferable, form.)

Suppose now that $P$ and $Q$ are nonzero finitely generated projective modules over a $k$-algebra $R$, and that we adjoin to $R$ a map from $P$ to $Q$ and a left inverse thereto, obtaining the universal extension ring

$$
S=R\left\langle i: \bar{P} \rightarrow \bar{Q}, j: \bar{Q} \rightarrow \bar{P} ; j i=1_{\bar{P}}\right\rangle
$$


Then $i j$ will be an idempotent endomorphism of $\bar{Q}$, resulting in a decomposition $\bar{Q}=Q_{1} \oplus Q_{2}$, and the maps $i$ and $j$ give an isomorphism between $P$ and $Q_{1}$. Hence let us define a fundamental $S$-module as one of the form $\bar{M} \oplus\left(\bigoplus Q_{2}\right)$ ( $M$ an $R$-module).

The study of this construction could be carried out in the same way as the three previous. (We would find that the appropriate universal extension of the ring $R_{0}=k \times k \times k=e_{P} k+e_{Q} k+e_{U} k$ has the form $\mathrm{m}_{2}(k) \times k \times k$, made an $R_{0}$-ring by the map $(\alpha, \beta, \gamma) \mapsto\left(\alpha e_{11}+\beta e_{22}, \beta, \gamma\right)$, with the module-maps $i$ and $j$ given by $\left(e_{21}, 0,0\right)$ and $\left(e_{12}, 0,0\right)$.) However, it is more interesting to note that the construction can be reduced to a combination of two of those studied above:

THEOREM 5.4. The k-algebra $S=R\left\langle i: \bar{P} \rightarrow \bar{Q}, j: \bar{Q} \rightarrow \bar{P} ; j i=1_{\bar{P}}\right\rangle$ may be obtained by first forming the algebra $R^{\prime}=R\left\langle e: \bar{Q} \rightarrow \bar{Q} ; e^{2}=e\right\rangle$, then putting $S=R^{\prime}\left\langle i, i^{-1}: \bar{P} \cong \bar{Q}_{1}\right\rangle$. Every submodule of a fundamental S-module is isomorphic to a fundamental S-module. $S_{\oplus}$ (Fund $S$ ) is obtained from $S_{\oplus}(\operatorname{Mod} R)$ by adjoining one generator $\left[Q_{2}\right]$ and one relation, $[P]+\left[Q_{2}\right]$ $=[Q]$. For any $R$-module $M, \mathrm{~h} \operatorname{dim}_{S} \bar{M}=\mathrm{h} \operatorname{dim}_{R} M$, and globally, $\mathrm{r} g \mathrm{gl} \operatorname{dim} S$ $=\mathrm{r} g l \operatorname{dim} R$, unless $\mathrm{r} \mathrm{gl} \operatorname{dim} R=0$, in which case $\mathrm{r} \mathrm{gl} \operatorname{dim} S \leqslant 1$.

Proof. That $S$ may be constructed as described is immediate by universal properties. (Put $j=i^{-1} \bar{e} ; e=i j$.) The homological and global dimension results follow immediately from Theorems 5.1 and 5.2 above.

The fundamental $S$-modules, as we have defined them here, are not all the fundamental modules of $S=R^{\prime}\left\langle i, i^{-1}: \bar{P} \cong \bar{Q}_{1}\right\rangle$, as defined for Theorem 5.2; rather, they are the subclass constructed by taking for $R^{\prime}$-module $M$ only fundamental $R^{\prime}$-modules (as defined for Theorem 5.1). Now note that when the construction $S=R^{\prime}\left\langle i, i^{-1}: \bar{P} \cong \bar{Q}_{1}\right\rangle$ is reduced to a coproduct over a ring $R_{0}^{\prime}$, the class of fundamental $R^{\prime}$-modules is $R_{0}^{\prime}$-stable. Hence replacing the applications of [7, Theorem 2.2 and Corollary 2.8] in the proof of Theorem 5.2, above, by [7, Corollaries 2.10, 2.11], we see, with the help of Theorem 5.1, that the class of fundamental $S$-modules is closed under submodules and that $S_{\oplus}$ (Fund $S$ ) has the form asserted.

Consider the following generalization of the above construction, which is sometimes useful. Let $R$ be a $k$-algebra, and $P_{1}, \cdots, P_{n}$ nonzero finitely generated projective $R$-modules, and $A$ any nonempty subset of the index-set $\{1, \cdots, n\}$. Let us adjoin to $R$ in a universal manner maps $f_{1}: \bar{P}_{1} \rightarrow \bar{P}_{2}$, $\cdots, f_{n}: \bar{P}_{n} \rightarrow \bar{P}_{1}$; and for all indices $a \in A$ (but no others!) let us set the cyclic product $f_{a-1} \cdots f_{2} f_{1} f_{n} \cdots f_{a}: \bar{P}_{a} \rightarrow \bar{P}_{a}$ equal to $1_{\bar{P}_{a}}$. Then for any $a, a^{\prime} \in A$, the products $f_{a^{\prime}-1} \cdots f_{a+1} f_{a}: \bar{P}_{a} \rightarrow \bar{P}_{a^{\prime}}$ and $f_{a-1} \cdots$ $f_{a^{\prime}+\mathrm{I}} f_{a^{\prime}}: \bar{P}_{a^{\prime}} \rightarrow \bar{P}_{a}$ will be inverses to one another, making $\bar{P}_{a} \cong \bar{P}_{a^{\prime}}$. Let us 
denote the common isomorphism class of the $\bar{P}_{a}(a \in A)$ by $\left[P^{\prime}\right]$.

On the other hand, for $b \notin A$, the map $e_{b}=f_{b-1} \cdots f_{b+1} f_{b}: \bar{P}_{b} \rightarrow$ $\bar{P}_{b}$ will be idempotent (because $A$ is nonempty, and for any $a \in A$, the product $e_{b}^{2}$ contains the subproduct $f_{a-1} \cdots f_{a}$, which can be deleted). Hence it induces a direct-sum decomposition $\bar{P}_{b}=P_{b}^{\prime} \oplus Q_{b}$. Since the corresponding cyclic product for $a \in A$ is $1_{\bar{P}_{a}}$, let us define $P_{a}^{\prime}=\bar{P}_{a}, Q_{a}=0$ for $a \in A$.

It is straightforward to show that the maps $f_{m}(m \in\{1, \cdots, n\})$ respect the decompositions $\bar{P}_{m}=P_{m}^{\prime} \oplus Q_{m}$. This means they take the form

$$
f_{m}=\left(\begin{array}{ll}
i_{m} & 0 \\
0 & g_{m}
\end{array}\right): P_{m}^{\prime} \oplus Q_{m} \rightarrow P_{m+1}^{\prime} \oplus Q_{m+1}
$$

where $i_{m}$ is an isomorphism, in fact, $i_{m-1} \cdots i_{m}=1_{\bar{P}_{m}^{\prime}} \quad(m=1, \cdots, n)$. The $g_{m}$ are homomorphisms $Q_{m} \rightarrow Q_{m+1}$, hence can be nontrivial only when neither $m$ nor $m+1$ lies in $A$, so that $Q_{m}$ and $Q_{m+1}$ are both nonzero.

One now easily verifies that our algebra $S=R\left\langle f_{m}: \bar{P}_{m} \rightarrow \bar{P}_{m+1} \quad(m=\right.$ $\left.1, \cdots, n) ; f_{a-1} \cdots f_{a}=1_{\bar{P}_{a}}\right\rangle$ can equivalently be obtained by the following steps:

(1) For all $b \in\{1, \cdots, n\}-A$, adjoin to $R$ universal idempotents $e_{b}$ : $\bar{P}_{b} \rightarrow \bar{P}_{b}$.

These give decompositions $\bar{P}_{b}=P_{b}^{\prime} \oplus Q_{b}$. For $a \in A$, we define $P_{a}^{\prime}=$ $P_{a}, Q_{a}=0$.

(2) For $m=1, \cdots, n-1(\mathrm{NB})$, adjoin isomorphisms $i_{m}, i_{m}^{-1}: P_{m}^{\prime}$ $\cong P_{m+1}$. (We may also define $i_{n}=i_{1}^{-1} i_{2}^{-1} \cdots i_{n-1}^{-1}: P_{n}^{\prime} \stackrel{\sim}{\rightarrow} P_{1}^{\prime}$.)

(3) For all $b$ such that neither $b$ nor $b+1$ lies in $A$, freely adjoin a map of nonzero projective modules: $g_{b}: Q_{b} \rightarrow Q_{b+1}$.

(For other $m$, we may formally write $g_{m}=0$. Thus we can now define $f_{m}=i_{m}+g_{m}: \bar{P}_{m} \rightarrow \bar{P}_{m+1}$ for all $m$, to establish the correspondence with our original description of $S$.)

Hence, by a finite number of applications of Theorems 5.1-5.3, one can establish properties of this construction quite analogous to the other results we have proved; in particular, the usual homological and global dimension formulae, and the description of $S_{\oplus}$ (Fund $S$ ) as obtained from $S_{\oplus}(\operatorname{Mod} R)$ by equating the elements $\left[P_{a}\right](a \in A)$ to a single value $\left[P^{\prime}\right]$, and adjoining new generators $\left[Q_{b}\right]$ and relations $\left[\bar{P}_{b}\right]=\left[Q_{b}\right]+\left[P^{\prime}\right]$ for all $b \notin A$.

Note that in all the constructions considered in this section, the result "submodules of fundamental modules are fundamental" implies in particular, that all projective $S$-modules are fundamental. Hence the structure of $S_{\oplus}(P-\operatorname{Mod} S)$ is obtained from that of $S_{\oplus}(P-M O d R)$ by the same construction that gives $S_{\oplus}($ Fund $S)$ from $S_{\oplus}(\operatorname{Mod} R)$. 
In the results of this section we have assumed throughout that the projective $R$-modules $P$ and $Q$ we were operating on were nonzero, so that the structure of $R_{0}$-ring ( $R_{0}=k \times k \times k$ etc.) that we got on $R$ after our reduction would be faithful, and the results of [7] would apply.

Suppose we try each of these constructions with $P$ and/or $Q$ the zero module. In the constructions of adjoining a universal idempotent endmorphism of $P$, or a universal map $P \rightarrow Q$, or, for the case $P=0$, of embedding $P$ universally as a direct summand in $Q$, we simply get $S=R$. The properties of $S$ then differ in a few minor ways from those stated in Theorems 5.1, 5.3, and 5.4 , but there is clearly no difficulty in describing these properties!

On the other hand, if we adjoin a universal isomorphism of a projective module $\boldsymbol{P}$ to the zero module, or a universal embedding of $\boldsymbol{P}$ as a direct summand in the zero module, this is equivalent to the less well understood construction $R\langle\bar{P}=0\rangle(\S 11)$.

I have not attempted to cram into Theorems 5.1-5.4 all the information one could get from [7] about these constructions, but only to illustrate the method, and set down enough information to permit some interesting examples in the next section. One can also get from Theorem 2.3 of [7] information on . homomorphisms among fundamental modules possibly more important for homological investigations than the superficial structure results described here. From Proposition 2.1 of [7] one gets descriptions of the $R$-module structures of fundamental $S$-modules, from which one can in turn get analogs of [7, Corollaries 2.10, 2.11]. Using the normal form for elements of a coproduct [7, Proposition 4.1; and §9] one can also get results such as the following:

AdDendum 5.5 to Theorem 5.3. The universal map $f: \bar{P} \rightarrow \bar{Q}$ is 1-1. More generally, given any two $R$-modules $U$ and $V$, the map

$$
\operatorname{Hom}(Q, V) \otimes_{k} \operatorname{Hom}(U, P) \rightarrow \operatorname{Hom}(\bar{U}, \bar{V})
$$

given by $a \otimes b \rightarrow \bar{a} \circ f \circ \bar{b}$ is $1-1$.

Finally, one can apply these methods to many more universal constructions.

6. Applications and examples. We mentioned earlier three classes of universal module map algebras that have been studied previously:

$$
\begin{aligned}
& V_{m, n}=k\left\langle i, i^{-1}: \overline{k^{n}} \cong \overline{\left.k^{m}\right\rangle},\right. \\
& U_{m, n}=k\left\langle i: \overline{k^{n}} \rightarrow \overline{k^{m}}, j: \overline{k^{m}} \rightarrow \overline{k^{n}} ; j i=1_{\overline{k^{n}}}\right\rangle, \\
& W_{n}=k\left\langle e: \overline{k^{n}} \rightarrow \overline{k^{n}} ; e^{2}=e\right\rangle .
\end{aligned}
$$


Theorems 5.1, 5.2 and 5.4 now yield immediately:

THEOREM 6.1. For $k$ a field and $n, m$ positive integers, the rings $U_{m, n}$, $V_{m, n}$ and $W_{n}$ are hereditary. For each of these rings $S$, let $I \in S_{\oplus}(P-M \circ d S)$ denote the isomorphism class $[S]$ of the free module of rank 1 . Then as additive semigroups with 0 , the $S_{\oplus}(P-M \circ d S)$ have, respectively, the following presentations:

$$
\begin{array}{rlrl}
U_{m, n} & : \text { generators } I,[P] ; & & \text { relation } m I=n I+[P] . \\
V_{m, n}: \text { generator } I ; & & \text { relation } m I=n I . \\
W_{n}: \text { generators } I,[P],[Q] ; & \text { relation } n I=[P]+[Q] .
\end{array}
$$

In particular, $U_{m, n}$ is an $(m-1)$-fir, $W_{n}$ an $(n-1)$-fir, and $V_{m, n}$ an $\inf (m-1, n-1)$-fir, and if $m=n$, a fir (!).

(Various parts of the above result were already known. Leavitt and Cohn determined the structures of the semigroups of free modules over $V_{m, n}$ and $U_{m, n}[25],[13]$. Cohn proved the last sentence of the above theorem, except for the fact that $V_{n, n}$ is a fir, in [13] and [15]. The only result that showed some of these rings to be hereditary, and determined the structures of all projective modules, is Skornyakov [30], for $V_{1,2} \cdot\left({ }^{3}\right)$ )

In fact, extending the spirit of the above constructions, we find that the semigroups $S_{\oplus}(P-M O d R)$ can be just about tailor-made:

THEOREM 6.2. Let $k$ be a field, and $A$ be a finitely generated abelian semigroup with a distinguished element $I \neq 0$, such that:

(i) $(\forall x, y \in A)(x+y=0) \Rightarrow(x=y=0)$, and

(ii) $(\forall x \in A)(\exists y \in A, n \geqslant 0), x+y=n I$.

Then there exists a k-algebra $R$ which is right and left hereditary, such that $S_{\oplus}(P-M \circ d R) \cong A$ as semigroups with distinguished element $I . R$ can be taken to have the weak universal property that for any k-algebra $S$ and any homomorphism $\phi$ of $A$ into $S_{\oplus}(P-M o d S)$, sending $I$ to $[S]$, there will exist a (generally nonunique!) k-algebra homomorphism $f: R \rightarrow S$ such that the map induced by $\_\otimes_{R} S$ from $S_{\oplus}(P-M o d R) \cong A$ to $S_{\oplus}(P-M \circ d S)$ is precisely $\phi$.

Proof. Choose a finite set of nonzero generators for $A$, of the form $\left\{I, p_{1}, q_{1}, \cdots, p_{r}, q_{r}\right\}$, with the property that for each $i, p_{i}+q_{i}=n_{i} I$ for some $n_{i}>0$. Any finitely generated abelian semigroup is finitely related (for the semigroup ring of a free abelian semigroup of finite rank is Noetherian!), nonzero powers will form a strictly ascending chain of submodules. Further, if

(3) A proof for $V_{2,3}$ is alleged in the same paper, but is incorrect. 
so we may choose a finite set of relations

$$
u_{j}=v_{j} \quad(j=1, \cdots, s),
$$

defining $A$ in terms of these generators. Dropping any relations of the form $0=0$, we see from hypothesis (i) that each $u_{j}$ and each $v_{j}$ will be a nonvacuous sum of $p$ 's, $q$ 's and $r$ s.

To construct $R$ now, adjoin to $k$, by $r$ successive applications of the construction of Theorem 5.1, idempotent matrices

$$
e_{i}: \bar{k}^{n_{i}} \rightarrow \bar{k}^{n_{i}} ; \quad e_{i}^{2}=e_{i} \quad(i=1, \cdots, r)
$$

giving decompositions $\bar{k}^{n_{i}}=P_{i} \oplus Q_{i}$. Then impose by $s$ applications of the construction of Theorem 5.2 the relations (4) among the $\left[P_{i}\right],\left[Q_{i}\right]$ and $I$. The assertion that the resulting $k$-algebra $R$ is hereditary and the description of $S_{\oplus}(P-M o d R)$ follow immediately from Theorems 3.1 and 3.2.

To see the weak universal property, let $S$ and $\phi$ be as in the statement thereof. For $i=1, \cdots, r$, choose a module-decomposition $S^{n_{i}}=P_{i}^{S} \oplus Q_{i}^{S}$ where $\left[P_{i}^{S}\right]=\phi\left(p_{i}\right),\left[Q_{i}^{S}\right]=\phi\left(q_{i}\right)$, and let $e_{i}^{S}$ be the projection of $S^{n_{i}}$ onto $P_{i}^{S}$ along $Q_{i}^{S}$. Likewise choose isomorphisms $a_{j}^{S}(j=1, \cdots, s)$ between the direct sums of copies of $P_{i}^{S}, Q_{i}^{S}$ and $S$ corresponding to the formal sums $u_{i}$ and $v_{i}$ in (4). By the universal module mapping property of $R$ these induce a homomorphism of $k$-algebras, $f: R \rightarrow S$, with the desired properties.

A particular case is:

EXAMPLE 6.3. There exists by Theorem 6.2 a right and left hereditary ring $R$ such that $S_{\oplus}(P-M \circ d R)$ is isomorphic to the additive semigroup $A$ of rational numbers generated by $\left\{1,1 \frac{1}{2}\right\}$, the free module of rank 1 corresponding to $1 \in A$.

Such a ring $R$ will be an integral domain satisfying condition $N$ of A. A. Klein [24], but which cannot be embedded in, or even mapped homomorphically into, any skew field.

The first assertion is clear. As to the meaning of the second, recall that Klein [24] observes that a necessary condition for the embeddability of a ring $R$ in a skew field is:

$\mathrm{N}$ : If $m$ is a positive integer, and $C$ a nilpotent $m \times m$ matrix over $R$, then $C^{m}=0$.

This was the key to his example [23] (discussed on pp. 67-68 below) answering Malcev's question on the embeddability of rings in skew fields.

Now if $C$ is a nilpotent $m \times m$ matrix over any ring $R$, consider it as a nilpotent endomorphism of the module $R^{m}$, and note that the kernels of its 
$R$ is right semihereditary, each of these kernels will be a direct summand in $R^{m}$ (because the image of the map in question is projective), hence each will be a direct summand in the next. So if $n$ is the least integer such that $C^{n}=0$, we get a decomposition of $R^{m}$ into a direct sum of $n$ nonzero submodules.

If $R$ is the ring described in the first assertion of Example 6.3, such a decomposition corresponds to an expression of $m$ as a sum of $n$ nonzero members of $\{1,112, \cdots\}$. By the order properties of the real numbers, this is possible only if $n \leqslant m$. So the order of nilpotence of $C$ is $\leqslant m$, so $R$ does indeed satisfy Klein's condition.

$R$ is also a domain, because left multiplication by a nonzero zero-divisor would similarly lead to a direct-sum decomposition of the free module of rank 1 .

But now suppose that this ring $R$ could be mapped into a skew field $K$. Apply the functor $S_{\oplus}($ P-Mod_ $)$ to the maps $k \rightarrow R \rightarrow K$. $S_{\oplus}(P-\operatorname{Mod} K)$ is isomorphic to the additive semigroup $\mathbf{N}$ of nonnegative integers, so we get maps $\mathbf{N} \rightarrow S_{\oplus}(P-M o d R) \rightarrow \mathbf{N}$ composing to the identity, i.e., a retraction of the semigroup $\left\{0,1,1 \frac{1}{2}, \cdots\right\}$ onto $N$, which clearly does not exist. In concrete terms, let $P$ be the projective $R$-module corresponding to $1 \frac{1 / 2}{2} \in A$. Then $P \oplus P \cong R^{3}$. Tensoring over $R$ with any $R$-sfield $K$, we would get a $K$-vector space $V$ such that $V \oplus V$ has dimension 3, which is impossible.

(We shall show elsewhere, using results of P. M. Cohn, that a semihereditary ring $R$ can be mapped homomorphically into a skew field if and only if the natural map $\mathbf{N} \rightarrow S_{\oplus}(P-M o d R)$ has a left inverse $\phi$, and embedded in a skew field if and only if $\phi$ can be taken to have zero "kernel": $\phi^{-1}(0)=\{0\}$.)

Back to generalities about $S_{\oplus}(P-M o d R)$ :

THEOREM 6.4. If in Theorem 6.2, the assumption that $A$ be finitely generated is dropped, the conclusion holds with "hereditary" weakened to "semihereditary".

Proof. As before, except that we now make a transfinite (well-ordered) sequence of universal constructions. At each limit ordinal, we take the direct limit ring. Direct limits respect $S_{\oplus}($ P-Mod_), and the property of being semihereditary (but not that of being hereditary). Each universal construction-step also respects the property of being semihereditary-this follows by applying to finitely generated ideals the fact that submodules of fundamental modules are isomorphic to fundamental modules.

This suggests:

Question 6.5. What restrictions, if any, are imposed on $S_{\oplus}(P-M \circ d R)$ ( $R$ a k-algebra), by the condition that $R$ be hereditary? Are any additional restrictions imposed by asking that $R$ have the weak universal property of Theorems 6.2 and 6.4 ? 
For commutative $R$, being hereditary definitely does restrict $S_{\oplus}(P-M o d R)$. To see this, note that if $P, Q \in P-M \circ d$, then the support of $P$ in $\operatorname{Spec} R$, an open-closed subset, will contain the support of $Q$ if and only if $Q$ can be written as a direct summand in a direct sum of copies of $P$. Hence from the structure of $S_{\oplus}(P-M O d R)$ one can determine the partially ordered set of openclosed subsets of Spec $R$. This will be a lattice with unique complements, so one gets in turn its structure of Boolean algebra, which can be identified with the Boolean algebra $B(R)$ of idempotent elements of $R$. But a necessary condition for $R$ to be hereditary is that $B(R)$ be hereditary [3, Theorem 4.4]. There exist nonhereditary Boolean algebras $B$ (and these can in fact be realized in the form $B(R)$ with semihereditary commutative $R$, e.g., $R=B$ ), so the condition that $R$ be hereditary does indeed nontrivially restrict $S_{\oplus}(P-\operatorname{Mod} R)$ in the commutative case.

Returning to noncommutative.$R$, and to Theorem 6.4 , note that in many non-finitely-generated cases we can still show the $R$ of that theorem to be hereditary. For example, suppose $A$ is a pushout over the nonnegative integers, $\mathbf{N}$, of a family of finitely generated semigroups:

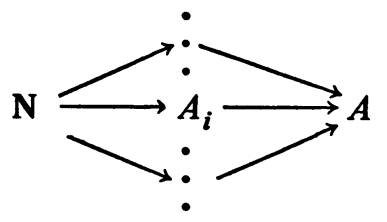

Then $R$, if constructed as above in terms of an appropriate set of generators and relations, will be the coproduct over $k$ of the corresponding rings for the $A_{i}$, hence, by Theorem 5.2 and the results of [7], hereditary. Starting with a direct product $k^{n}$ in place of $k$, and using matrix-reductions as in the preceding section, we can get pushouts of still more general diagrams.

For another example, note that the universal ring for the semigroup generated by $1 / n$ (with distinguished element 1 ) is (if appropriately constructed) $m_{n}(k)$. Hence a universal ring for the additive group of nonnegative rational numbers is given by the direct limit of the system:

$$
k \rightarrow \mathrm{m}_{2}(k) \rightarrow \mathrm{m}_{6}(k) \rightarrow \cdots \rightarrow \mathrm{m}_{n !}(k) \rightarrow \cdots
$$

(where the map $\mathfrak{m}_{n !}(k) \rightarrow \mathfrak{m}_{(n+1) !}(k)$ is given by applying $\mathfrak{m}_{n !}$ to the map $\left.k \rightarrow \mathrm{m}_{n+1}(k)\right)$. Now in a von Neumann regular ring, a countably generated right ideal is projective, hence a countable direct limit of semisimple artin rings is hereditary; in particular, the ring described above is hereditary.

The universal ring for the semigroup defined by generators $I, p_{i}, q_{i}(i=$ $0,1, \cdots)$ and relations 


$$
p_{0}=I, \quad p_{i}=p_{i+1}+q_{i}(i=0,1, \cdots)
$$

is similarly the direct limit of the system:

$$
k \rightarrow k \times k \rightarrow k \times(k \times k) \rightarrow k \times k \times(k \times k) \rightarrow \cdots,
$$

and so hereditary for the same reason. It is, in fact, the ring of continuous $k$ valued functions on the 1-point compactification of the natural numbers. If we replace this compact space by the spectrum of any other countable Boolean algebra, we get a similar example.

On the other hand, some semigroups which we do not know whether one can realize as $S_{\oplus}(P-M \circ d R)$ for hereditary $R$ are: the additive group of nonnegative real numbers; $\{0\} \cup$ \{rational numbers $\geqslant 1\}$, and the semigroup of continuous integer-valued functions on the spectrum of a nonhereditary Boolean ring.

The results of $\S 5$ throw an interesting light on the theorem that a free associative algebra over a field $k$ is a fir. Such an algebra is a coproduct of copies of the polynomial ring $k[x]$, so the results of [7] make this a consequence of the fact that $k[x]$ is a principal ideal domain. We all can prove this in our sleep by the Euclidean algorithm; but we can now obtain this result, alternatively, by describing $k[x]$ as $k\langle x: \bar{k} \rightarrow \bar{k}\rangle$. In effect, the proof of Theorem 5.3 has reduced this module-theoretic question about $k[x]$ to one about the still simpler ring $\mathrm{t}_{2}(k)$ !

Of course, the proof of Theorem 5.3 uses the results of [7], which are based on heavy computational machinery which is ultimately descended from the Euclidean algorithm (cf. [7, §3]).

7. Endomorphism-ring constructions and the left adjoint of $m_{n}$. Let $R$ be a $k$-algebra, and $P$ a finitely generated projective $R$-module. Then by Theorem 3.3, for any $k$-algebra $B$ we can construct an $R$-ring $k, S$, with a universal homomorphism of $k$-algebras, $H: B \rightarrow \operatorname{End}_{S} \bar{P}$. (We adjoin to $R$ an endomorphism of $P$ for each element of $B$, and the appropriate relations among these. This means taking for $A$ and $B$ in that theorem 1-object categories, where the object of $A$ has endomorphism ring $k$ and maps to $P$ in $P-M O d R$, and the object of $B$ has endomorphism ring $B$.)

This make $\bar{P}$ a $(B, S)$-bimodule ${ }_{k}$ (the subscript $k$ here meaning that right and left multiplication by elements of $k$, via $B$ and $S$, agree). Hence we get a functor $\otimes_{B} \bar{P}$ taking the category of right $B$-modules to the category of right $S$-modules. For any $B$-module $N$, let $\widetilde{N}$ denote the $S$-module $N \otimes_{B} \bar{P}$. As usual, for $M$ an $R$-module, $\bar{M}$ will denote $M \otimes_{R} S$. We shall call an $S$ module of the form $\bar{M} \oplus \widetilde{N}$ ( $M$ an $R$-module, $N$ a $B$-module) a fundamental $S$-module. Note that $\widetilde{B} \cong \bar{P}$. 
THEOREM 7.1. Over the k-algebra $S=R\langle B \rightarrow$ End $\bar{P}\rangle$, every submodule of a fundamental module is isomorphic to a fundamental module. The semigroup $S_{\oplus}($ Fund $S)$ may be constructed as a pushout over $\mathrm{N}$, the nonnegative integers:

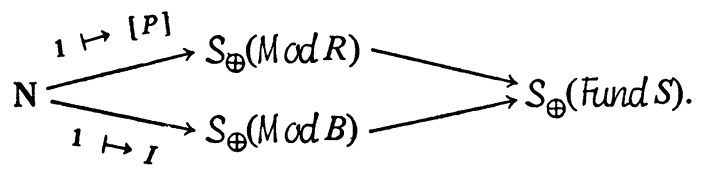

For all R-modules $M, \mathrm{~h} \operatorname{dim}_{S} \bar{M}=\mathrm{h} \operatorname{dim}_{R} M$, and for all B-modules $N$, $\mathrm{h} \operatorname{dim}_{S} \tilde{N}=\mathrm{h} \operatorname{dim}_{B} N$. Globally, $\mathrm{r} \mathrm{gl} \operatorname{dim} S=\max (\mathrm{r} \mathrm{gl} \operatorname{dim} R, \mathrm{r} \mathrm{gl} \operatorname{dim} B)$, unless the right-hand side is 0 , in which case $\mathrm{r} \mathrm{gl} \operatorname{dim} S \leqslant 1$.

Suppose $M$ is a finitely generated $R$-module not having any direct summand isomorphic to $P$. Then any fundamental S-module which is a homomorphic image of $\bar{M}$ can be written $\bar{M}^{\prime}$, where $M^{\prime}$ is some homomorphic image of $M$. For such an $M, \bar{M} \cong \bar{M}^{\prime} \Leftrightarrow M \cong M^{\prime}$.

Proof. As in $\S 5$, we first reduce by the matrix-ring construction to the case where $P$ is a proper direct summand in $R: R=P \oplus U=e_{P} R+e_{U} R$. Putting $R_{0}=e_{P} k+e_{U} k \cong k \times k$, we find that $R_{0}\left\langle B \rightarrow\right.$ End $\left.\bar{P}_{0}\right\rangle=B \times k$, so $S=R \Perp_{R_{0}}(B \times k)$.

A standard module over this coproduct algebra will have the form $\left(M \otimes_{R} S\right)$ $\oplus\left(L \otimes_{B \times k} S\right)$, where $M$ is an $R$-module and $L$ a $(B \times k)$-module. The first summand is of the form $\bar{M}$, as desired. To analyze the second, note that a $(B \times k)$-module $L$ will be a direct sum of a $B$-module $N$ and a $k$-module $V$, each made a $(B \times k)$-module in the obvious manner. Another way of writing this is $L=\left(N \otimes_{B} X\right) \oplus\left(V \otimes_{k} Y\right)$, where $X$ denotes $B$, made a $(B, B \times k)$ bimodule by the natural left $B$-module structure and the right $(B \times k)$-module structure of $e_{P}(B \times k)$, and $Y$ denotes $k$ with the natural left $k$-module structure and the right structure $e_{U}(B \times k)$. Hence when we go on to tensor over $B \times k$ with $S$, we get $\left(N \otimes_{B} \bar{P}\right) \oplus\left(V \otimes_{k} \bar{U}\right)$. The first summand has the form $\widetilde{N}$, as desired, while the second is a direct sum of copies of $\bar{U}$, and so can be thrown into the summand $\bar{M}$. This proves that the standard $S$-modules as in [7] are here again precisely the fundamental $S$-modules.

The results of the first paragraph of the theorem follow easily.

The last paragraph follows from [7, Theorem 2.3(q.v.)]: If $M$ has no direct summand isomorphic to $P$, then the only sort of basic transfer one can perform on $\bar{M}$ will involve copies of $\bar{U} \cong U_{0} \otimes_{R_{0}} S$. But over $B \times k, U_{0}$-induced basic modules play a very simple role in the module theory, and we see that in the construction of the cited theorem, basic transfers can in this case be eliminated; and the result follows.

Now suppose that for $\boldsymbol{P}$ in the above theorem we take the free module 
of rank $n$. We may write the resulting ring as $S=R\left\langle B \rightarrow m_{n}\right\rangle$ (since for any ring $A$, End $\left(A^{n}\right)=\mathrm{m}_{n}(A)$ ). As the free $R$-module $R^{n}$ is induced by a $k$ module, $k^{n}$, Theorem 3.4 yields:

Proposition 7.2. $R\left\langle B \rightarrow \mathrm{m}_{n}\right\rangle \cong R \Perp_{k} k\left\langle B \rightarrow \mathrm{m}_{n}\right\rangle$.

So for the rest of this section we shall examine the construction $k\left\langle B \rightarrow \mathrm{m}_{n}\right\rangle$, which we shall call $\mathfrak{w}_{n}(B)$. This will be a $k$-algebra $S$ with a universal map of $B$ into its $n \times n$ matrix ring; in effect, a universal coefficient ring for $n \times n$ matrix representations of $B$.

Our taking $P$ free also allows a simplification in the construction of the ring given in the proof of Theorem 7.1: we can drop the module $U$ (cf. parenthetical remark after proof of Theorems 5.1-5.3, p. 46. Doing so, and showing the matrix reduction involved in that proof explicitly, we find that $R\left\langle B \rightarrow \mathfrak{m}_{n}\right\rangle=\mathfrak{M}_{n}^{-1}\left(\mathfrak{m}_{n}(R) \Perp_{k} B\right)\left(\mathbb{M l}_{n}^{-1}\right.$ as defined in $\left.\S 4\right)$, and in particular, $\mathfrak{w}_{n}(B)=k\left\langle B \rightarrow \mathrm{nt}_{n}\right\rangle=\mathscr{J} \sum_{n}^{-1}\left(\mathfrak{m}_{n}(k) \Perp_{k} B\right)$.

Note that the universal property of $\mathfrak{m}_{n}$ says that it is the left adjoint to the functor $\mathrm{m}_{n}: \operatorname{Ring}_{k} \rightarrow \operatorname{Ring} g_{k}$. Recall (p. 41 above) that $\mathrm{m}_{n}$ factors:

$$
\operatorname{Ring}_{k} \stackrel{\mathfrak{N}_{n}}{\longrightarrow} \mathrm{m}_{n}(k)-\operatorname{Ring}_{k} \stackrel{\boldsymbol{S}_{n}}{\longrightarrow} \text { Ring }_{k} \text {. }
$$

Now it is easy to see that the forgetful functor $\mathcal{J}_{n}$ has the left adjoint $\mathfrak{m}_{n}(k) \Perp_{k-}: \operatorname{Ring} g_{k} \rightarrow \mathfrak{m}_{n}(k)-R$ ing $_{k}$, while since $\mathfrak{M}_{n}$ is an equivalence of categories, its inverse will be its left adjoint. The adjoint of a composition is the composition of the adjoints (when these exist), thus "explaining" the formula $\mathfrak{w}_{n}\left(\mathcal{)}=\mathcal{M l}_{n}^{-1}\left(\mathfrak{m}_{n}(k) \Perp_{k}\right)\right.$.

Let us now apply Theorem 7.1 to this construction $\mathfrak{w}_{n}$. The " $R$ " of that theorem is now $k$, so we shall replace " $B$ " by $R$. Since all $k$-modules are free, the fundamental $S$-modules have the form $F \oplus \widetilde{N}$, where $F$ is a free $S$-module and $N$ an $R$-module. But $S^{n}=\bar{P} \cong \widetilde{R}$, hence we can transfer copies of $S^{n}$ from $F$ to $\widetilde{N}$, and write the most general fundamental $S$-module as $\widetilde{N} \oplus S^{m}$ $(m<n)$.

In the last assertion of the theorem, the possible choices for $M$ are precisely the $k$-vector spaces of ranks $m<n$. It thus says that any fundamental homomorphic image of a module $S^{m}(m<n)$ has the form $S^{m^{\prime}}\left(m^{\prime} \leqslant n\right)$, and that the rank $m$ of $S^{m}$ is unique. Since in particular, a homomorphic image of $S^{m}$ in a free $S$-module is fundamental, this says that $S$ is an $(n-1)$-fir. Summarizing, we have:

TheOREM 7.3. Let $R$ be a k-algebra, $n$ a positive integer, and $S$ the $k$-algebra $\mathfrak{w}_{n}(R)=k\left\langle R \rightarrow \mathfrak{m}_{n}\right\rangle$. Then every submodule of a fundamental $S$ module $\widetilde{N} \oplus S^{m}$ is isomorphic to a fundamental S-module. The semigroup 
$S_{\oplus}($ Fund $S)$ can be obtained from $S_{\oplus}(\operatorname{Mod} R)$ by adjoining one generator $[S]$, and one relation $[\widetilde{R}]=n[S]$, (i.e., $\widetilde{R} \cong S^{n}$. Of course, $[S]$ is the "distinguished element" of this semigroup, though that of $S_{\oplus}(\operatorname{Mod} R)$ was $[R])$. For all R-modules $N, \mathrm{~h} \operatorname{dim}_{S} \widetilde{N}=\mathrm{h} \operatorname{dim}_{R} N$, and globally, $\mathrm{r} \mathrm{gl} \operatorname{dim} S$ $=\mathrm{rgl} \operatorname{dim} R$, unless $\mathrm{rgl} \operatorname{dim} R=0$, in which case $\mathrm{rgl} \operatorname{dim} S \leqslant 1$.

Last but not least, $S$ is an $(n-1)$-fir.

The constructions described above overlap those considered in $\S \S 5$ and 6 . Specifically, they include those cases in which only one projective module is involved: thus, $R\left\langle e: \bar{P} \rightarrow \bar{P} ; e^{2}=e\right\rangle$ can be described as $R\langle k \times k \rightarrow$ End $P\rangle$, and the $P=Q$ cases of the other constructions similarly reduce. In particular in Theorem 6.1, we have $W_{n}=\mathfrak{w}_{n}(k \times k), V_{n, n}=\mathfrak{w}_{n}\left(k\left[x, x^{-1}\right]\right)$, and $U_{n, n}$ $=\mathfrak{w}_{n}(k(x, y ; x y=1))$.

One can use the preceding results on further universal constructions, e.g., adjoining to a ring $R$ two universal commuting $n \times n$ matrices (or more generally, endomorphisms of a finitely generated projective module $P$ ), etc. However, we shall make some other sorts of applications in the next section.

The interested reader will have no difficulty in proving:

ADDENDUM 7.4 TO THEOREM 7.3. If, for some integer $i, R$ is an $(i-1)$ fir, then $\mathfrak{w}_{n}(R)$ is an $(n i-1)$-fir. If $R$ is not an i-fir, then $\mathfrak{w}_{n}(R)$ is not an ni-fir.

FURTHER Remarks. We described $\mathfrak{w}_{n}(R)$ as a "universal coefficient ring" for $n \times n$ matrix representations of $R$. A "universal coefficient ring" would seem to be a good way, generally, to look at the left adjoint of any representable functor (cf. [22], [11]) with domain the category of associative rings or $k$ algebras. For instance, the formal power series functor $\mathfrak{p}: \operatorname{Ring}_{k} \rightarrow \operatorname{Rin} g_{k}$ is representable, and so by universal algebra (not by results of this paper) it has a left adjoint, associating to a $k$-algebra $R$ a $k$-algebra $S=k\langle R \rightarrow \mathfrak{p}\rangle$, characterized as having a universal map $R \rightarrow p(S)$. What homological or other good properties this construction may have, we do not know. Some examples already have names: the left adjoint to the commutator-brackets functor from associative algebras to Lie algebras is called the (Poincaré-Birkhoff-Witt) universal enveloping algebra construction. The left adjoint to the group-of-units construction $\operatorname{Ring}_{k} \rightarrow$ Group is the group-algebra construction, $G \mapsto k[G]$. If we compose this functor and adjoint with $\mathfrak{m}_{n}$ and its adjoint $\mathfrak{w}_{n}$ respectively, we see that the left adjoint to $\mathrm{GL}_{n}: \operatorname{Ring}_{k} \rightarrow$ Group is the construction $G \mapsto$ $\mathfrak{w}_{n}(k[G])$, providing a universal $k$-algebra for $n$-dimensional representations of any group $G$.

Amitsur in [1] associates to a $k$-algebra $R$ a commutative $k$-algebra $C$ 
with a universal map $R \rightarrow \mathfrak{m}_{n}(C)$. We see that this ring will in fact be $c\left(\mathfrak{w}_{n}(R)\right)$, where $c$ is the abelianization functor (quotient-by-the-commutator-ideal, the left adjoint of the inclusion $\operatorname{Comm} R \operatorname{Ring}_{k} \rightarrow R$ ing $_{k}$ ). However since the functor $c$ has, as far as I know, no nice homological properties, we can deduce nothing, from the present results, about this construction.

If $R$ is a $k$-algebra, then homomorphisms of $R$ into $n \times n$ matrix-rings over sfields $k_{k}$ correspond to homomorphisms of $\mathfrak{w}_{n}(R)$ into sfields $k_{k}$. P. M. Cohn has used this observation in his work on noncommutative algebraic geometry. ([17, pp. 55-58]. He calls $\mathfrak{w}_{n} M_{1 / n}$ )

8. Applications: "Integrity is no defense", or "Everything you've wanted to know about integral domains but were afraid to ask". By integral domain we will mean ring without zero-divisors, not necessarily commutative. Recall [16] that 1-fir = integral domain. L. Small [31] (improving a result of Chase) gives an example of an algebra $R$ over an arbitrary field $k$ which is right hereditary but not left semihereditary; in fact, not even left 1-hereditary. (A ring is called right or left $\alpha$-hereditary, for a cardinal $\alpha$, if all right, resp. left ideals generated by $\alpha$ elements are projective as modules.) By Theorem 7.3 (and its right-left dual), $\mathfrak{w}_{n}(R)$ for any positive integer $n$ will be a $k$-algebra which is right hereditary but not left semihereditary, (in fact, not left $n$-hereditary) and which will also be an $(n-1)$-fir. In particular, taking $n>1$, we get:

EXAMPLE 8.1. An integral domain which is right but not left semihereditary.

The moral of this example is that being an integral domain, or even an $n$ fir, is no protection against most homological pathologies! Explicitly:

COROLlaRY 8.2. Suppose $\mathrm{P}$ is a property of algebras over a field $k$, such that if $R$ satisfies $\mathrm{P}$, so does $R \Perp_{k} \mathrm{~m}_{n}(k)$, and such that if $\mathrm{m}_{n}(R)$ satisfies $\mathrm{P}$, so does $R$ (or more generally, such that in the latter case $R$ will satisfy a related condition $\left.\mathrm{P}_{n}\right)$. Then if there is any k-algebra $R$ satisfying $\mathrm{P}$, there is an $(n-1)$-fir, namely $\mathfrak{w}_{n}(R)$, satisfying $\mathrm{P}\left(\right.$ resp. $\left.\mathrm{P}_{n}\right)$. In particular, taking any $n>1$, we get an integral domain satisfying $\mathrm{P}$ (resp. $\left.\mathrm{P}_{n}\right)$ ).

Here is a similar application, answering a question raised by J. C. Robson:

EXAMPLE 8.3. A right and left hereditary integral domain with an infinite ascending chain of idempotent 2-sided ideals. Let $k$ be a field, and $R$ the $k$ algebra of continuous $k$-valued functions on the one-point compactification of the positive integers. Then $R$ is hereditary, as observed on p. 54 above, and clearly has an infinite ascending chain of idempotent ideals. These properties are respected by $\mathfrak{m}_{n}$ (any $n$ ), and by the results of [7], ${ }_{-}{ }_{k} \mathfrak{m}_{n}(R)$ likewise respects the properties of being right and left hereditary. Further, note that if $I$ is any ideal of a $k$-algebra $R$ ( $k$ a field), $R^{\prime}$ another $k$-algebra, and $S=R \Perp_{k} R^{\prime}$, 
then $(R / I) \Perp_{k} R^{\prime} \cong S / S I S$. Since $R / I$ embeds in $(R / I) \Perp_{k} R^{\prime}$, the map $I \mapsto$ SIS from the partially ordered set of ideals of $R$ to the partially ordered set of ideals of $S$ is $1-1$. Finally, for two ideals $I, J \subseteq R$, we have $(S I S)(S J S) \supseteq$ SIJS, so if $I$ is idempotent, so is $S I S$. It follows that for $R$ as above, and any $n>1, \mathfrak{w}_{n}(R)$ will satisfy the desired conditions.

One can list a few more properties of this example: The free module of rank $n$ will have an infinite ascending chain of direct summands, $P_{1} \subseteq P_{2}$ $\subseteq \cdots$, such that the chain of ideals described above are in fact the trace ideals $T_{P_{1}} \subseteq T_{P_{2}} \subseteq \cdots ;$ and the quotients of the ring by these ideals are again hereditary, in fact, isomorphic to the original ring.

One can deduce from Corollary 7.5 that if $\mathbf{P}$ is a condition which is satisfied by some $k$-algebra, respected in the manners indicated by $-\Perp m_{n}(k)$ and $m_{n}(-)$, and also preserved by ultraproducts, then there exists a semifir satisfying P. But I know no application of this observation.

9. The $n$-term weak algorithm for universal rings; with applications. To show that $U_{m, n}$ is an $(m-1)$-fir, and similar results for $V_{m, n}$ and $W_{n}$, P. M. Cohn in fact showed that these rings satisfied $r$-term weak algorithm, for appropriate values of $r$ [13], [15]. He presented in general form the conditions needed for this argument in Theorem 3.1 of [15]. We shall use this theorem to get fairly general hypotheses under which a $k$-algebra defined by a $k$-linear universal mapping property-as in Theorem 3.3, but with $R=k$-will satisfy $n$-term weak algorithm.

This rather long and technical section can be skipped by those not interested; it is not required for anything else in this paper.

If $k$ is a commutative ring and $A$ is a $k$-linear category, we shall call an object $X \in \mathrm{Ob}(\mathrm{A})$ right isolated if, for all $Y \neq X, \operatorname{Hom}(Y, X)=0$, left isolated if, for all $Y \neq X, \operatorname{Hom}(X, Y)=0$. Let us call $X$ a seed if it is right and left isolated, and $\operatorname{Hom}(X, X) \cong k$. Note that in the context of the next theorem, to require that an object $X$ of $A$ be a seed is to forbid ourselves to impose on $R$ relations on the constructed module homomorphisms that involve any maps already in P-Mod $k$ and having range or domain $F(X)$, except for $1_{F(X)}$. This is, indeed, a condition frequently satsified in the constructions we have considered. In particular, for many of these constructions, all objects of $A$ satisfy condition (a) below. Since the proof of Theorem 9.1 is more complicated when we must also consider case (b), we shall first prove the theorem assuming that only (a) occurs, and give a number of applications of this case, then return to the proof and show how to handle (b) as well.

THEOREM 9.1. Let $k$ be $a$ field, $A$ and $B$ small $k$-linear categories, and $F: A \rightarrow$ P-Mod $k$ and $G: A \rightarrow B$ k-linear functors, such that $G$ yields a bijection of objects, $\mathrm{Ob} A \leftrightarrow \mathrm{Ob} B$. 
Suppose $n$ is a positive integer such that for every $X \in O b(A)$, either:

(a) $X$ is a seed in $A$ and $F(X) \in P$-Mad $k$ has dimension $>n$, or

(b) $G(X)$ is left or right isolated in $B, G: \operatorname{Hom}_{A}(X, X) \rightarrow \operatorname{Hom}_{B}(G(X), G(X))$ is surjective, and $F(X) \neq 0$.

Then the universal $k$-algebra $R=k\langle B\rangle$ is either 0 , or satisfies $n$-term weak algorithm with respect to an appropriate filtration. (The condition for $R$ to be nonzero, and the form of the filtration, are given in the proof.)

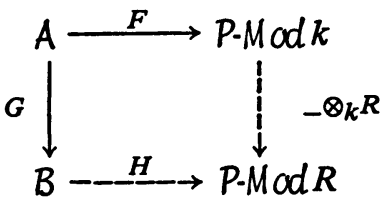

Proof (Assuming all objects satisfy (a)). Clearly, if for some $X \in$ $\mathrm{Ob}(\mathrm{A})$, $\operatorname{Hom}(G(X), G(X))$ is the zero ring, then $k\langle B\rangle=0$. Hence let us assume Hom $(G(X), G(X))$ is a nonzero $k$-algebra for all $X$; then we shall show that $R$ satisfies $n$-term weak algorithm. (The condition for $R \neq 0$ will be less trivial in the general case of the theorem.)

For each $X \in \mathrm{Ob}(\mathrm{A})$, let $d(X)>n$ denote the dimension of the vector space $F(X) \in P$-Mod $k$. Then we can identify $F(X)$ with the vector space $\mathbf{c}_{d(X)}(k)$ of column vectors of height $d(X)$ over $k$ (by choosing a basis). Thus, $\operatorname{Hom}_{\mathrm{p}-\operatorname{Mad} k}(F(X), F(Y))$ is the space of $d(Y) \times d(X)$ matrices over $k$. For any $k$-algebra $R$, we can likewise identify $F(X) \otimes_{k} R$ with $c_{d(X)}(R)$, and $\operatorname{Hom}_{\mathrm{P}-M_{\mathrm{Od}} R}(F(X) \otimes R, F(Y) \otimes R)$ with the set of $d(Y) \times d(X)$ matrices over $R$.

Thus, the $k$-linear functor $H: B \rightarrow P-M \circ d R$ which we wish to study will consist of $k$-linear maps from the vector spaces $\operatorname{Hom}_{B}(G(X), G(Y))$ into these spaces of matrices over $R$. So to get a description of our universal $R$, let us begin by choosing, for each $X, Y \in O b(A)$ a $k$-basis $S(X, Y)$ for $\operatorname{Hom}_{B}(G(X), G(Y))$. When $X=Y$ we require that this basis contain $1_{G(X)}$ (assumed $\neq 0): S(X, X)=T(X, X) \cup\{1\}$. For $X \neq Y$ we define $T(X, Y)$ $=S(X, Y)$.

Now let $\Theta$ be the free associative $k$-algebra on a set of symbols:

$$
t_{i j} \quad(i \leqslant d(Y), j \leqslant d(X) ; t \in T(X, Y) ; X, Y \in O b(A)) .
$$

(Here $t_{i j}$ will eventually represent the $(i, j)$ component of the matrix $H(t)$.) Let us also define

$$
\left(1_{G(X)}\right)_{i j}=\delta_{i j} \in \Theta \quad(i, j \leqslant d(X) ; X \in O b(A)) .
$$

Thus, we have now defined $s_{i j} \in \Theta$ for each $s$ in the basis $S(X, Y)$ of each $\operatorname{Hom}_{B}(G(X), G(Y))$ and each appropriate $i, j$. We can now extend by 
$k$-linearity to get maps:

$$
\left.\begin{array}{rl}
f & \mapsto f_{i j} \\
\operatorname{Hom}_{B}(G(X), G(Y)) & \rightarrow \Theta
\end{array}\right\} \quad(i \leqslant d(Y), j \leqslant d(X)) .
$$

Or, fitting these $f_{i j}$ into $d(X) \times d(Y)$ matrices, and identifying these with homomorphisms of modules of column vectors, we get, for each $X, Y \in \mathrm{Ob}(\mathrm{A})$, a map:

$$
\begin{aligned}
f & \mapsto\left(\left(f_{i j}\right)\right) \\
\operatorname{Hom}_{B}(G(X), G(Y)) & \mapsto \operatorname{Hom}_{\rho-M_{\alpha d} \Theta}\left(c_{d(X)}(\Theta), c_{d(Y)}(\Theta)\right) .
\end{aligned}
$$

These maps are $k$-linear, and take identity elements to identity matrices. We shall now show how to divide out the free algebra $\Theta$ by relations saying that these maps also respect composition; over the quotient ring $R$, the maps $f \mapsto\left(\left(\bar{f}_{i j}\right)\right)$ will define a functor $B \rightarrow$ P-Mod $R$, with the desired universal property.

For our $k$-linear maps of Hom-spaces to respect composition, it will clearly suffice that they do so on our basis-sets, i.e., that:

$$
\left(\left(s_{i j}\right)\right)\left(\left(s_{i j}^{\prime}\right)\right)=\left(\left(\left(s s^{\prime}\right)_{i j}\right)\right) \quad\left(s \in S(Y, Z), s^{\prime} \in S(X, Y) ; X, Y, Z \in O b(A)\right) .
$$

Because our maps already send identity elements of $S(X, X)$ to identity matrices, the above equation holds automatically when $s$ and/or $s$ is an identity element, so it suffices to impose the above relations for elements $t \in T(Y, Z)$, $t^{\prime} \in T(X, Y)$. Expanding the above matrix-equations into equations in elements, we get:

$$
\begin{aligned}
\sum_{i<d(Y)} t_{h i} t_{i j}^{\prime}=\left(t t^{\prime}\right)_{h j} \quad(h \leqslant d(Z), & j \leqslant d(X) ; t \in T(Y, Z), \\
t^{\prime} & \in T(X, Y) ; X, Y, Z \in O b(A)) .
\end{aligned}
$$

Note that in the above equation, $t t^{\prime} \in \operatorname{Hom}_{B}(G(X), G(Z))$ is in general not a member of the basis $T(X, Z)$, so the expression on the right-hand side of (7) represents a $k$-linear combination of elements $t_{h j}^{\prime \prime}\left(t^{\prime \prime} \in T(X, Z), h, j\right.$ fixed in (7)), and possibly a scalar term arising from (5).

We now define $R$ to be the factor-ring of the free algebra $\Theta$ obtained by imposing the relations (7). We shall continue to write $t_{i j}$ for the images in $R$ of the generators $t_{i j}$ of $\Theta . R$ will indeed be the universal $k$-algebra of our theorem, the functor $H$ being given by (6). We shall now prove by [15, Theorem 3.1] that $R$ satisfies $n$-term weak algorithm with respect to the filtration defined by $v(a)=$ minimum degree of expressions for $a$ in terms of the generators $t_{i j}$ (each $t_{i j}$ being taken of degree 1).

We must first put the generators and relations for $R$ in the form needed 
for that theorem. Here $n+1$ will take the place of that theorem's $n \cdot\left({ }^{4}\right)$ For the family of generators there called $\left(x_{i v}\right)$, we take the $t_{i \nu}$ with $\nu \leqslant$ $n+1$ (thus, the index-set $I$ of that theorem is here $U_{X, Y} d(Y) \times T(X, Y)$. Since $d(X)>n$ by hypothesis, $\nu$ always has the full range $1, \cdots, n+1$ as required). Similarly, for the family $\left(y_{\nu j}\right)$ (not required to be disjoint from $\left(x_{i \nu}\right)$ ) we take the $t_{\nu j}$ with $\nu \leqslant n+1$, and for the family $\left(z_{h}\right)$, we take remaining generators, the $t_{i j}$ with $i, j>n+1$. The defining relations (7) then take the form [15, (3.5)] if we bring all terms with $i>n+1$ to the right.

We must now demonstrate the existence of the $n+1$ normal forms required by that theorem. Fix any $\mu \in\{1, \cdots, n+1\}$. Recall that the $\mu$ th normal form is obtained by bringing to the right in our defining relations all but the $\mu$ th term of the summation; so that (7) becomes:

$$
t_{h \mu} t_{\mu j}^{\prime}=\left(t t^{\prime}\right)_{h j}-\sum_{i \leqslant d(Y), i \neq \mu} t_{h i} t_{i j}^{\prime} \quad(h, j \text { etc. as in (7)). }
$$

We use these formulas to reduce any expression in the given generators to a $k$-linear combination of monomials which involve no subwords $t_{h \mu} t_{\mu j}^{\prime}$ with $t \in T(Y, Z), t^{\prime} \in T(X, Y)$. (Subwords $t_{h \mu} t_{\mu j}^{\prime}$ with $t \in T(Y, Z), t^{\prime} \in T\left(X, Y^{\prime}\right)$, $Y \neq Y^{\prime}$ are allowed.) Indeed, any application of a formula $\left(7_{\mu}\right)$ to a monomial $w$ in our generators will reduce it to a linear combination of monomials $w^{\prime}$ each having either shorter length than $w$, or the same length but fewer "bad"' subwords, so the process will eventually yield an expression with no such subwords. To show that this expression is independent of the choices we make of which subword to reduce when, it suffices, by $[8$, Theorem $1,(a) \Rightarrow(b)]$, to check that for any word formed from two "overlapping" words as on the lefthand side of $\left(7_{\mu}\right)$, the results of the two ways of reducing them can themselves be reduced to a common value. The words we must test are clearly:

$$
\begin{array}{r}
t_{g \mu} t_{\mu \mu}^{\prime} t_{\mu j}^{\prime \prime} \quad\left(t \in T(Y, Z), t^{\prime} \in T(X, Y), t^{\prime \prime} \in T(W, X) ;\right. \\
g \leqslant d(Z), j \leqslant d(W)) .
\end{array}
$$

The fact that the two possible reductions of this term reduce in turn a common value will follow from the associative law in $B$; to be precise, from the equation $t\left(t^{\prime} t^{\prime \prime}\right)=\left(t t^{\prime}\right) t^{\prime \prime}$. One could carry out this calculation by brute force, but we can simplify it by adding to (8) some further terms which we know do reduce unambiguously. (Cf. $\left[8\right.$, Theorem $\left.1,\left(\mathrm{a}^{\prime}\right) \Rightarrow(\mathrm{b})\right]$.) Indeed, consider instead of (8) the sum:

$$
\sum_{h, i} t_{g h} t_{h i}^{\prime} t_{i j}^{\prime \prime}
$$

in which all terms except the one in question are uniquely reducible. Now if, on the one hand, we reduce the first pair of factors in all terms of the above sum

$\left({ }^{4}\right)$ The theorem in question establishes $(n-1)$-term weak algorithm. The $\lambda(R)$ in the statement of the theorem is the least $m$ for which $m$-term weak algorithm does not hold. 
having $h=\mu$ (which in particular involves one of the possible reductions of the term (8)), we see from $\left(7_{\mu}\right)$ that all degree-3 terms will cancel, and we will get:

$$
\sum_{i}\left(t t^{\prime}\right)_{g i} t_{i j}^{\prime \prime}
$$

If we now in this expression reduce all terms with $i=\mu$ we get: $\left(8^{\prime \prime \prime}\right)$

$$
\left(t t^{\prime} t^{\prime \prime}\right)_{g i}
$$

It is clear that by the same procedure, but starting from the right, we will get the same results; so we have proved the existence of the normal form with respect to the index $\mu$.

It remains only to show that the normal form for each index $\mu$ satisfies the condition $N_{\mu}$ of $[15, \mathrm{p} .273]$. It is easy to see (even in the general case of [15]) that to verify this condition, it suffices to v srify it with $f, g$, and $h$ taken to be members of the given set of generators. The condition then becomes: If $f, g$, and $h$ are generators, such that the word $f g$ is $\mu$-reduced, while the word $g h$ has reduced form $[g h]_{\mu}$, then the degree-3 terms of $f[g h]_{\mu}$ are $\mu$-reduced. This is clearly true-the point being that given $f$, the irreducibility of a product $f \cdot y$, where $y$ is an irreducible monomial with leftmost factor $t_{i j}(t \in T(X, Y))$ depends only on $i$ (whether it equals $\mu$ ), and the range-object $X$ (whether it equals the domain-object of the rightmost factor of $f$ ). But we see from $\left(7_{\mu}\right)$ that all highest-degree terms resulting from a reduction have the same "left-hand data" as did the original word!

So [15, Theorem 3. $]$ now tells us that $R$ has $n$-term weak algorithm, completing the proof of Theorem 9.1 when all $X \in A$ satisfy (a).

As applications we have:

THEOREM 9.2 Let $k$ be a field, $R$ a $k$-algebra, and $n$ a positive integer. Then the k-algebra $\mathfrak{w}_{n}(R)$ satisfies $(n-1)$-term weak algorithm with respect to an appropriate filtration $v$.

Proof. $\mathfrak{w}_{n}(R)$ is the case of the construction of Theorem 9.1 in which A consists of a single seed $X$, mapped by $F$ to $k^{n} \in P$-Mod $k$, and $B$ consists of a single object $G(X)$ with $\operatorname{Hom}_{B}(G(X), G(X))=R$.

(I can prove by other methods the analog for this theorem of Addendum 7.4 above, namely: if $R$ satisfies $(i-1)$-term weak algorithm with respect to some filtration, then $\mathfrak{w}_{n}(R)$ satisfies $(n i-1)$-term weak algorithm with respect to an appropriate induced filtration.)

We similarly get:

THEOREM 9.3. Suppose $A$ is a semigroup satisfying the conditions of Theorem 6.4 (i.e., like Theorem 6.2 but not necessarily finitely generated), and suppose that for some positive integer $n, A$ can be presented in such a way 
that in all defining relations where the element I occurs, it occurs with coefficient $>n$. Then the k-algebra $R$ of that theorem, constructed using such a presentation, will satisfy n-term weak algorithm.

PROOF. Though in proving Theorems 6.2 and 6.4 we did the construction in a number of steps, it is not hard to see that the resulting universal ring $R$ has the form $k\langle B\rangle$ for a $B$ (and $A, F, G$ ) having all the properties required by the case of Theorem 9.1 that we have proved.

The algebras $U_{m, n}, V_{m, n}$ and $W_{n}$ of $\S 6$ are examples of this construction, hence the first two satisfy $\min ((m-1),(n-1))$-term weak algorithm, and the last, $(n-1)$-term weak algorithm. But in fact, one of the original applications of the method of [15, Theorem 3.1] was to show that $U_{m, n}$ satisfies $(m-1)$ term weak algorithm, regardless of $n .\left(^{5}\right)$

Indeed, if we look at the generators $a_{i j}(i \leqslant m, j \leqslant n)$ and $b_{i j}(i \leqslant n$, $j \leqslant m$ ) and relations $\Sigma_{i<m} b_{h i} a_{i j}=\delta_{h j}$ defining this algebra, we see that we never sum over a subscript with range $n$, so the fact that some subscripts have such a range imposes no limitation on the number of normal forms we can obtain-we can write our relations in the form $\left(7_{\mu}\right)$ for $\mu=1, \cdots, m$ even if $n<m$.

To incorporate this fact, that we do not have any relations on the sums $\Sigma_{i<n} a_{h i} b_{i j}$, into a presentation of $U_{m, n}$ as a universal mapping algebra $k\langle B\rangle$ (which will necessarily be a different presentation from the one which only gave us $\min ((m-1),(n-1))$-term weak algorithm), we set up the categories $A$ and $B$ so that the range of the map to be represented by $\left(\left(a_{i j}\right)\right)$ and the domain of the map to be represented by $\left(\left(b_{i j}\right)\right)$ are distinct objects of $B$. Thus:

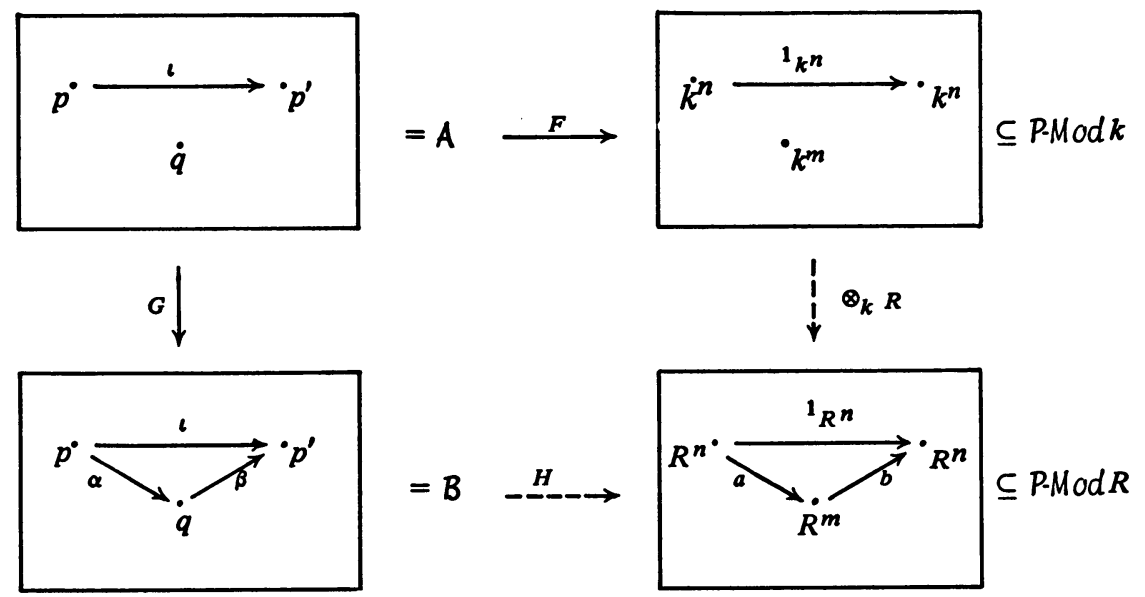

(5) For the next few paragraphs, $m$ and $n$ denote the subscripts in $U_{m, n}$, so the $n$ of Theorem 9.1 corresponds to $m-1$. 
(If we consider the dot indicating an object also to represent its identity map, then the dots and arrows in our pictures of $A$ and $B$ are meant to give $k$-bases for these categories; while in P-Mod $k$ and P-Mod $R$ we only show the images of $A$ and $B$. The triangles in the lower two diagrams are commutative.)

The property of this diagram that now insures that we do not sum over an index having range $n$ is precisely the fact that $p$ and $p^{\prime}$ satisfy condition (b) in the statement of Theorem 9.1. The "right or left isolated" part insures that we nowhere introduce both generators mapping into and generators mapping out of such an object, and the surjectivity of $\operatorname{Hom}_{A}(X, X) \rightarrow \operatorname{Hom}_{B}(G(X), G(X))$ insures that we do not introduce any endomorphisms of these objects.

Note that for objects $X$ which satisfy condition (b), the statement of Theorem 9.1 does not require $X$ to be a seed in $A$-and indeed, we use the map l: $p \rightarrow p^{\prime}$ in the construction of $U_{m, n}$ above.

Note also that in the description of $U_{m, n}$, since we never sum over the index $n$, we need not even think of it as a matrix index. That is, instead of defining $R$ by one $m \times n$ and one $n \times m$ matrix of generators, and a relation assigning a value to their product, we could have introduced $n m \times 1$ and $n 1 \times m$ matrices, and $n^{2}$ relations on their $1 \times 1$ products. Thus we may replace the " $n$-dimensional" objects $p$ and $p^{\prime}$ of $A$ by "one-dimensional" objects, and increase the number of generating maps by a factor of $n$. This idea will be used in the proof below to obtain an initial simplification. It is really unnecessary for a case like the above, where the corresponding objects of $A, p$ and $p^{\prime}$, have trivial endomorphism rings, but its value in the general case will be, precisely, to eliminate complexities arising from the consideration of these rings.

Proof of Theorem 9.1: General case. Partition the objects of $A$ into three disjoint sets: $A_{(a)}$, consisting of objects satisfying condition (a) of the hypothesis, $A_{(r)}$, consisting of objects satisfying (b) whose images under $G$ are right isolated in $B$, and $A_{(l)}$ consisting of objects satisfying (b) and having left isolated images. (If an object is eligible for more than one of these sets, make the choice arbitrarily.)

For $X \in A_{(r)}, \quad Y \in A_{(l)}$ we know nothing about $\operatorname{Hom}(X, X), \operatorname{Hom}(X, Y)$, or $\operatorname{Hom}(Y, Y)$. (All other Hom-sets, or at least their images in $B$, are determined by our hypothesis; in particular, all Hom-sets in $A$ involving objects of $A_{(a)}$.) Therefore, let us simplify things by defining a new $k$-linear category $A^{\prime}$, having the same objects as $A$, and the same Hom-sets except that the Hom-sets of the above three types are replaced by the sets of matrices,

$$
\text { Hom }_{\mathrm{P}-\operatorname{Mad} k}(F(X), F(X)), \text { Hom }_{\mathrm{P}-\operatorname{Med} k}(F(X), F(Y)),
$$

and

$$
\operatorname{Hom}_{P-\operatorname{Med} k}(F(Y), F(Y)) \text {. }
$$


The functor $F$ factors through $A^{\prime}$ in an obvious natural way (diagram below). We define $B^{\prime}$ as the pushout (among $k$-linear categories with object set $O b A=O b B=O b A^{\prime}$ ) of the maps of $A$ into $B$ and $A^{\prime}$ :

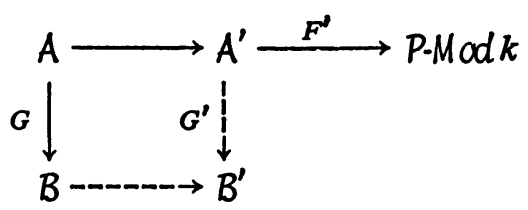

It is not hard to see that the universal $k$-algebra for the new diagram

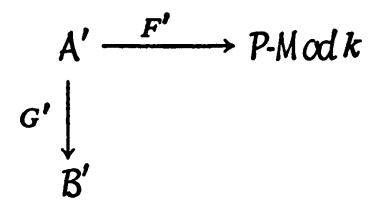

will be the same as for

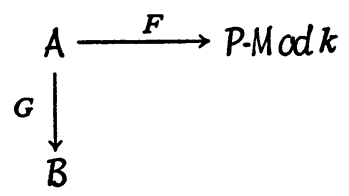

The new diagram will also still satisfy the hypotheses of our theorem, assuming the old one did. But it may happen that the new functor, $G^{\prime}: A^{\prime} \rightarrow B^{\prime}$, is not faithful. In this case, it is easy to deduce that our universally constructed algebra $R$ will be the zero ring. Henceforth, we will assume $G^{\prime}$ faithful, and prove that $R$ has $n$-term weak algorithm.

Let $X$ be any object of $A_{(r)}^{\prime}$. Because

$$
\begin{aligned}
\operatorname{Hom}_{A^{\prime}}(X, X) & \cong \operatorname{Hom}_{B^{\prime}}\left(F^{\prime}(X), F^{\prime}(X)\right) \\
& \cong \operatorname{Hom}_{P-M_{a d} k}\left(G^{\prime}(X), G^{\prime}(X)\right)=\mathfrak{m}_{d(X)}(k),
\end{aligned}
$$

a full matrix ring, the object $X$ will behave in the $k$-linear category $A^{\prime}$ like a direct sum of $d(X)$ copies of some object $X^{\prime}$, and likewise its images $F^{\prime}(X), G^{\prime}(X)$ will behave like direct sums of $d(X)$ copies of an object. (Indeed, $F^{\prime}(X)$ is a direct sum of $d(X)$ copies of $k$ in P-Modk.) Hence, let us modify our categories $A^{\prime}$ and $B^{\prime}$ by first adjoining for each $X$ an object $X^{\prime}$ to $A^{\prime}$ and $G^{\prime}\left(X^{\prime}\right)$ to $B^{\prime}$, such that $X$ and $G^{\prime}(X)$ become $d(X)$-fold direct sums of $X^{\prime}$ and $G^{\prime}\left(X^{\prime}\right)$, then delete the original objects $X$ and $G(X)$. (We omit the tedious description of exactly how this is done. The idea is: look at the sets $\operatorname{Hom}(X, Y)$ etc. as right $\mathfrak{m}_{d(X)}(k)$-modules, and apply the functor $\mathfrak{r} \frac{-1}{d(X)}$ to get the $k$-module $\operatorname{Hom}\left(X^{\prime}, Y\right)$.) We do this for all $X \in A_{(r)}^{\prime}$, then do the same thing for all $Y \in A_{(l)}^{\prime}$. 
The result is a third pair of categories, $A^{\prime \prime}$ and $B^{\prime \prime}$, and functors $F^{\prime \prime}$ : $A^{\prime \prime} \rightarrow$ P-Mod $k, G^{\prime \prime}: A^{\prime \prime} \rightarrow B^{\prime \prime}$, yielding the same universal $k$-algebra $R$, and satisfying the original hypotheses of our theorem and also: For all $X \in A_{(r)}^{\prime \prime}$, $Y \in A_{(l)}^{\prime \prime}$,

$F(X)=F(Y)=k \in$ Ob P-Mod $k$;

$F$ is an isomorphism on $\operatorname{Hom}(X, X), \operatorname{Hom}(Y, Y)$ and $\operatorname{Hom}(X, Y)$;

$G$ is an isomorphism on $\operatorname{Hom}(X, X)$ and $\operatorname{Hom}(Y, Y)$, and is faithful on all of $A^{\prime \prime}$.

Note that for such $X$ and $Y, \operatorname{Hom}_{\mathrm{A}}(X, Y)$ will be 1-dimensional as a $k$-vector space, with a distinguished generator, $F^{-1}\left(1_{k}\right)$. The image of the generator under $G$ will be a nonzero element of $\operatorname{Hom}_{B}(G(X), G(Y))$, which we shall also call 1.

We now complete the proof as before: for each $X, Y \in A$, we choose a basis $S(X, Y)$ of $\operatorname{Hom}_{B}(G(X), G(Y))$. If $X=Y$ or if $X \in A_{(r)}, \quad Y \in A_{(l)}$, we take $S(X, Y)$ to have the form $T(X, Y) \cup\{1\}$; otherwise we define $T(X, Y)=S(X, Y)$. We set up generators and relations for $R$, and apply [15, Theorem 3.1] exactly as before.

In particular, we recover the result that the ring $U_{m, n}$ satisfies $(m-1)$ term weak algorithm.

The above theorem is also applicable to the rings constructed by A. A. Klein [23] and A. J. Bowtell [10] to answer Malcev's question of whether an integral domain whose multiplicative semigroup of nonzero elements is embeddable in a group is itself embeddable in a sfield. Klein's example was, in effect, $\mathfrak{w}_{n}\left(k[t] / t^{r}\right)$, where $2<n<r$. The $n \times n$ matrix $T$ over this ring corresponding to $t \in$ $k[t] / t^{r}$ is nilpotent $\left(T^{r}=0\right)$, but $T^{n} \neq 0$, which is impossible for an $n \times n$ matrix over a sfield. Bowtell's example is generated by the entries of an $r \times n$ matrix $X$ and an $n \times r$ matrix $Y$ with relations making $X Y$ diagonal (but not necessarily with equal diagonal values), $n$ and $r$ as above. The diagonal values will all be nonzero, hence if the ring lay in a sfield, we would have a nonsingular $r \times r$ matrix over a sfield which was a product of matrices of smaller size, which is again impossible.

Bowtell's proof that his ring has multiplicative semigroup embeddable in a group is based on showing that it is a graded ring with 2-term (in fact, $(n-1)$ term) weak algorithm. This is easily seen to be a case of Theorem 9.1 above. Klein gives a different proof of embeddability, but Bowtell's argument can also be applied to his ring: it has $(n-1)$-term weak algorithm by Theorem 9.2.

(Cohn [14, Theorem 5.2, Corollary 1] has more recently proved that any atomic 2 -fir $R$ can be embedded in a ring in which all nonzero elements of $R$ become invertible (cf. next section). In particular, any ring with 2-term weak algorithm, graded or not, has multiplicative semigroup embeddable in a group, so 
the rings $U_{n, r}, V_{n, r}$ give further counterexamples to Malcev's question. It seems likely that Cohn's result should extend to arbitrary 2-firs.)

With the help of the full form of Theorem 9.1, one would like to extend Theorem 9.3 to get $n$-term weak algorithm for rings associated with a larger class of semigroups $A$. Since the rings constructed are semihereditary, they will be $n$ firs if and only if the only representations of $n I$ as a sum of two elements in $A$ are as $m I+m^{\prime} I$, where $m+m^{\prime}=n$. For such a semigroup $A$ it would be nice to conclude that $R$ can also be taken to have $n$-term weak algorithm. (Of course, whether $R$ has $n$-term weak algorithm can depend on the presentation of $A$ from which it was constructed. For $A=$ the nonnegative integers, with one generator $I$ and no relations, we get $R=k$ which trivially has full weak algorithm. But presenting $A$ by the generator $I$ and the relation $n I=n I$, we get $V_{n, n}$, which is still a fir but can be shown to have $(n-1)$-term but not $n$-term weak algorithm.) The result that $U_{m, n}$ has $(m-1)$-term weak algorithm is of this form, but there are many cases for which I do not see how to get such a result. E.g., if $A$ has generators $I, p$ and relations:

$$
(n+1) I=n I+p, \quad p=2 p
$$

then any $R$ with the properties stated in Theorem 6.2 will be an $n$-fir, but I do not know whether such $R$ can be constructed to have $n$-term weak algorithm.

10. Localization. If $R$ is a $k$-algebra and $f: P \rightarrow Q$ a homomorphism of $R$-modules, we would like to study the constructions $S=R\left\langle\bar{f}^{-1}\right\rangle$ and $S=$ $R\left\langle g ; g \bar{f}=1_{\bar{P}}\right\rangle$. I have not been able to prove any good general results like those we got for the constructions studied in $\S \S 5,7$, and 9; but in this section we shall examine the good and bad properties of some classes of examples, and also indicate the equivalence of the general construction to certain special cases.

Consider first the construction $R\left\langle\bar{f}^{-1}\right\rangle$, when the $k$-algebra $R$ is commutative. The map $R \rightarrow R\left\langle\bar{f}^{-1}\right\rangle$ is an epimorphism (in the category-theoretic sense-not a surjective map!) because 2-sided inverses are unique. But an epimorph of a commutative ring is commutative [29], hence $R\left\langle\bar{f}^{-1}\right\rangle$ is again commutative, and, can be identified with the corresponding construction on commutative rings, $R\left[f^{-1}\right]$. In particular, taking $P=Q=$ the free module of rank 1 , we see that the adjunction of an inverse to an element of a commutative ring is a case of the construction we are considering.

(In the case of arbitrary $P, Q$ and $f, S$ can be obtained from $R$ by inverting a certain ideal which is locally principal on $\operatorname{Spec} R$ : the "generator" is taken to be 0 over those connected components of $\operatorname{Spec} R$ on which $P$ and $Q$ have different ranks, and constructed locally as a determinant elsewhere. Thus Spec $S$ is $\operatorname{Spec} R$ minus a closed subvariety. For $R$ an integral domain, $S$ 
is either 0 , or the localization obtained by inverting an invertible integral ideal of $R$.)

The process of localization on commutative rings is well known to have extremely good properties! If $S=R\left[f^{-1}\right]$, then every $S$-module $N$ can be written $\bar{M}$ for some $R$-module $M$; in fact, we can take $M=N$ (under restriction of scalars), but alternatively, we can take $M$ finitely generated or finitely presented as an $R$-module if $N$ is so as an $S$-module, by using the original generators and relations, and "clearing denominators". Any submodule of $\bar{M}$ is induced by a submodule of $M$.

$S$ will be flat as an $R$-module, hence for any $R$-module $M, \mathrm{hd}_{S} \bar{M} \leqslant$ $\mathrm{hd}_{R} M$. But this inequality can be embarrassing, for it may not be possible to lift an $S$-module $N$ to an $R$-module of the same homological dimension. In particular, $S$ may have projective modules not induced by any projective $R$-module. As a simple example, let $R$ be the commutative $k$-algebra defined by two generators $x$ and $y$, and one relation $x y=0$; the ring of polynomial functions on the union of the $x$ - and $y$-axes. Then in $S=R\left[(x+y)^{-1}\right]$, the point at which these axes are joined is deleted, and $S$ splits into a direct product, $R\left[x, x^{-1}\right] \times R\left[y, y^{-1}\right]$. In particular, the projective $S$-modules generated by $(1,0)$ and $(0,1)$ do not lift to projective $R$-modules.

A more subtle case, not based on zero-divisors, was pointed out to me by D. Eisenbud. Let $R=\mathbf{R}[x, y, z]$, where $\mathbf{R}$ is the field of real numbers, and let $M$ denote the submodule of $R^{3}$ given by $\{(a, b, c) \mid a x+b y+c z=0\}$. $M$ may be thought of as the module of polynomial vector-fields on 3-space that are everywhere perpendicular to the radius vector. It is easy to show that if $f \in$ $R$ is a polynomial with value zero at the origin, and we take $S=R\left[f^{-1}\right]$, then $M \otimes S$ is a projective $S$-module. But if $f$ is also chosen so that its zero-set has no intersection with the unit sphere $x^{2}+y^{2}+z^{2}=1$ (e.g., $f=x^{2}+y^{2}+$ $z^{2}$ ), then this projective module is not induced by any projective $R$-module because the induced vector-bundle on the sphere cannot be extended to $\mathbf{R}^{3}$.

Note, however, that if $R$ is hereditary, "projective $R$-module" is equivalent to " $R$-module embeddable in a free $R$-module", and one can deduce using the flatness of $S$ that the map $S_{\oplus}(P-\operatorname{Mod} R) \rightarrow S_{\oplus}(P-\operatorname{Mod} S)$ is indeed then surjective. Even then it is not generally injective-one of the uses of localization in commutative ring theory is to "cure" rings of nonfree projective modules!

In the case of noncommutative localization even hereditary rings may acquire new projectives. Let $R=k[x]$, a polynomial ring in one indeterminate, and let $S=R\langle y ; x y=1\rangle$, the ring obtained by adjoining a right inverse to $x$. This ring is $k\langle x, y ; x y=1\rangle=U_{1,1}$, which we know has semigroup of finitely generated projectives defined by generators $I$ and $p$, and the relation $I+p=$ I. The projective module represented by $p$ is the image of the idempotent ele- 
ment $1-y x$ of $S$. It is not induced by a member of $S_{\oplus}(P-M \circ d R)$-in fact, not by any member of $S_{\oplus}(\operatorname{Mod} R)$, though curiously, the dual projective left $R$-module $P^{*} \cong S(y x-1)$ is isomorphic to $S \otimes_{R}(R / R x)$.

This would appear to be a pathology characteristic of one-sided inverses, but a slight modification of this example shows two-sided inverses behaving in the same spirit. Let $R$ be the free associative algebra on two indeterminates, $k\langle x, y\rangle$, and let $S=R\left\langle(x y)^{-1}\right\rangle=k\langle x, y, z ;(x y) z=z(x y)=1\rangle$. If $x$ and $y$ commuted, this construction would be equivalent to adjoining inverses to $x$ and $y$, but here $x$ acquires only a right inverse, $y z$, and $y$ only a left inverse, $z x$; and $S$ has an idempotent that gives a projective not induced by $R: 1-$ $y z x$. In fact, this ring is an example of the construction discussed on pp. 47-48 We have adjoined a cycle of module-homomorphisms:

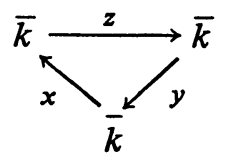

and relations saying that some, but not all of their cyclic products are identity maps. It is easy to verify from the discussion on those pages that $S_{\oplus}(P-\operatorname{Mod} S)$ is again determined by generators $I$ and $p$, and one relation $I+p=I .\left(^{6}\right)$ (The one thing which is better in this example than in the one-sided case is that the right and left projective modules $P$ and $P^{*}$ are both induced by $R$-modules $R / y R$ and $R / R x$ respectively.)

Finally, note that even in very nice cases, localization of noncommutative rings does not generally give flat extensions. (Localization by right or left Ore denominator sets, like commutative localization, does give right or left flat extensions. But it is not generally equivalent to universal localization-cf. [16, Exercise 7.2.11, p. 258].) E.g., let $R$ be the free associative algebra $k\langle x, y\rangle$, and $S=R\left\langle x^{-1}\right\rangle$. (Or more generally, let $S$ be any $R$-ring in which $x$ becomes invertible.) In $R$, the equation $a x=b y$ has no nonzero solution, but it does in $S: a=y x^{-1}, b=1$. Hence $S$ cannot be right flat over $R$, for $S \otimes_{R}$ does not preserve exactness of the sequence of left modules:

$$
0 \rightarrow R^{2} \stackrel{\left(\begin{array}{l}
x \\
y
\end{array}\right)}{\longrightarrow} R \text {. }
$$

There remain a few statements of the sort we proved for the constructions of $\S \S 5$ and 7 that might be true for these localization operations; namely:

(6) Perhaps this observation that adjoining a two-sided inverse to an element is not, without special hypotheses, "nicer" than adjoining a one-sided inverse can illuminate the fact that Malcev found it convenient to study the embedding problem for groups in semigroups in terms of separate adjunctions of right and left inverses [27], [12, VII. 3]. 
( $\alpha$ ) for the constructions of left and two-sided inverses, that every submodule of a fundamental right $S$-module $\bar{M}=M \otimes_{R} S$ be isomorphic to a fundamental module; and for all the constructions:

( $\beta$ ) that $\mathrm{h} \operatorname{dim}_{S} \bar{M} \leqslant \mathrm{~h} \operatorname{dim}_{R} M$ for $R$-modules $M$ (despite nonflatness of $S$ ), and

( $\gamma$ ) that $\mathrm{r} \mathrm{gl} \operatorname{dim} S \leqslant \mathrm{rgl} \operatorname{dim} R$ (or $\leqslant 1$ if $\mathrm{r} \mathrm{gl} \operatorname{dim} R=0$ ).

As with the constructions of $\S \S 5$ and 7 , the study of localization can be reduced to the study of a very simple type of coproduct-but in this case, one to which the results of [7] do not apply:

If $R$ is a $k$-algebra and $f: P \rightarrow Q$ a nonzero homomorphism of finitely generated projective $R$-modules, we can, in considering any questions about the category of $R$-modules, assume that $R=P \oplus Q \oplus U$ as modules, and make $R$ a faithful $\left(\mathrm{t}_{2}(k) \times k\right)$-ring $k_{k}$ via $\left(\left(\begin{array}{ll}\alpha & 0 \\ \beta & \gamma\end{array}\right), \delta\right) \rightarrow \alpha e_{P}+\beta f+\gamma e_{Q}+\delta e_{U}$. Now the universal extension of $t_{2}(k)$ in which $e_{21}$, as a map $e_{11} t_{2}(k) \rightarrow e_{22} t_{2}(k)$ is inverted is $\mathfrak{m}_{2}(k)$. The universal ring in which it is left inverted is $\mathfrak{m}_{2}(k) \times k$ under the map $\left(\begin{array}{ll}\alpha & 0 \\ \beta & \gamma\end{array}\right) \rightarrow\left(\left(\begin{array}{cc}\alpha & 0 \\ \beta & \gamma\end{array}\right), \gamma\right)$, and the universal ring in which it is right inverted is likewise $k \times \mathrm{m}_{\mathbf{2}}(k)$. Hence questions about the general constructions $R\left\langle\bar{f}^{-1}\right\rangle, R\left\langle g ; g \bar{f}=1_{\bar{P}}\right\rangle, R\left\langle g ; \bar{f} g=1_{\bar{Q}}\right\rangle$ are reduced to questions about coproducts over $\mathrm{t}_{2}(k) \times k$ of a $k$-algebra $R$ with $\mathfrak{m}_{2}(k) \times k$, respectively, $\mathrm{m}_{2}(k) \times k \times k$, respectively $k \times \mathrm{m}_{2}(k) \times k$ via the above mappings.

In the case of 2-sided inverses, one can make a neater reduction, which gets rid of the summand " $U$ " and thus the final factors " $-\times k$ " above. Namely, over the original $R$, choose $U$ as before such that $P \oplus Q \oplus U$ is free, then put $P^{\prime}=P \oplus P \oplus U$ and $Q^{\prime}=Q \oplus Q \oplus U$; and note that to invert $f$ is the same as to invert

$$
f^{\prime}=\left(\begin{array}{lll}
f & 0 & 0 \\
0 & f & 0 \\
0 & 0 & 1_{Q}
\end{array}\right): P^{\prime} \rightarrow Q^{\prime}
$$

But $P^{\prime} \oplus Q^{\prime}$ is free, so we can reduce by matrices to the case $R=P^{\prime} \oplus Q^{\prime}$, and then only have to take a coproduct over $t_{2}(k)$ with $m_{2}(k)$.

For many questions, such as that of homological dimension, one can use the results of $\S 5$ to make a more drastic reduction, for all three kinds of inverses. The key observation is that the adjunction to $R$ of universal isomorphisms $P \oplus$ $Q \cong R$ and/or $P \cong Q \cong R$ commutes with the universal inversion of $f$ and exactly preserves global dimension (except when $\mathrm{gl} \operatorname{dim} R=0$ ). The first such adjunction allows us to reduce, as above, to the case of a coproduct over $t_{2}(k)$, but without restriction on the type of inverse we are adjoining. In the second case, 
the left, right or two-sided inversion of $f: P \rightarrow Q$ becomes the left, right or two-sided inversion of an element of $R$. Let us record this result in the nicest case, that of 2-sided inversion:

LEMMA 10.1. If any of the following statements are true, all are:

(i) Given any k-algebra $R$ and any nonzero homomorphism $f: P \rightarrow Q$ of finitely generated projective $R$-modules, $\mathrm{r} \mathrm{gl} \operatorname{dim} R\left\langle\bar{f}^{-1}\right\rangle \leqslant \mathrm{r} \mathrm{gl} \operatorname{dim} R$.

(ii) Given any k-algebra $R$ and any nonzero element $x \in R$, $\mathrm{r} \mathrm{gl} \operatorname{dim} R\left\langle x^{-1}\right\rangle \leqslant \mathrm{r} \mathrm{gl} \operatorname{dim} R$.

(iii) Given any faithful $\mathfrak{t}_{2}(k)$-ring $k_{k}, R$, one has $\mathrm{rgl} \operatorname{dim} R \Perp_{\mathrm{t}_{2}(k)} \mathrm{m}_{2}(k)$ $\leqslant \mathrm{r}$ gl $\operatorname{dim} R$.

The one outstanding recent positive result about universal localization of which we are aware is the following. (Recall that Cohn [18] calls an $n \times n$ matrix full if it cannot be factored as an $n \times(n-1)$ times an $(n-1) \times n$ matrix; and a class $\Sigma$ of square matrices factor-closed if $A B \in \Sigma \Rightarrow A \in \Sigma$, $B \in \Sigma$.)

TheOREM (COHN [18]). Let $R$ be a semifir, and $\Sigma$ a factor-closed set of full square matrices over $R$. Then the map $R \rightarrow R\left\langle\Sigma^{-1}\right\rangle$ is an embedding and $R\left\langle\Sigma^{-1}\right\rangle$ is a semifir.

Note that the example $k\left(x, y,(x y)^{-1}\right)$ showed "bad" behavior precisely because of the failure of the set of elements being inverted to be factor-closed.

We wonder whether a version of the above theorem could be proved with $R$ assumed an $N$-fir, all matrices in $\Sigma$ assumed of size $\leqslant m \times m$, and the conclusion saying that $R\left(\Sigma^{-1}\right)$ is an $n$-fir, for some appropriate relation among the integers $N, n$ and $m$.

An example is given in $[5, \S 5]$ in which $R$ is a domain with polynomial identity, and $x$ a nonzero element of $R$, but the universal localization $R\left\langle x^{-1}\right\rangle$ cannot be a prime ring satisfying the same polynomial identities. Whether it fails to be prime, or fails to satisfy the same identities, or both, is an open question! More general kinds of localization definitely fail to preserve polynomial identities:

EXAMPLE 10.2 (AFTER IsBell, [22, p. 268]). A finite-dimensional $k$-algebra $R$ satisfying the identities of $2 \times 2$ matrices over commutative rings, having $a$ map $f: P \rightarrow Q$ of finitely-generated projective modules such that $R\left\langle\bar{f}^{-1}\right\rangle$ is infinite-dimensional, and satisfies no polynomial identities.

Construction. First note that it will suffice to find such an $R$ with some infinite-dimensional extension $S$ without polynomial identity, which is generated by (the coordinates of) an inverse to $\bar{f}$, for such an $S$ will be a 
homomorphic image of $R\left\langle\bar{f}^{-1}\right\rangle$, which will thus also have these properties. (In fact, the reader will not find it hard to show that the $S$ we shall display actually is $R\left\langle\bar{f}^{-1}\right\rangle$.)

Let $S$ denote $\mathrm{m}_{2}(k\langle x, y\rangle)$, where $k\langle x, y\rangle$ is the free associative algebra in 2 indeterminates over the field $k$, and let $R$ be the 5-dimensional subalgebra $\left(\begin{array}{cc}k & k+k x+k y \\ 0 & k\end{array}\right) \subseteq S$. In $R$, let $P=e_{11} R, Q=e_{22} R, f=e_{21}: P \rightarrow$ $Q$. In $S, \bar{f}=e_{21}: \bar{P} \rightarrow \bar{Q}$ has the 2 -sided inverse $e_{12}: \bar{Q} \rightarrow \bar{P}$. It is straightforward to verify that $R$ and $e_{21}$ together generate $S . R$ satisfies the identities of $2 \times 2$ matrices over commutative rings because it is isomorphic to the corresponding subalgebra of $\mathfrak{m}_{2}(k[x, y]) . S$ contains the subalgebra $k\langle x, y\rangle$, and hence is infinite-dimensional and satisfies no polynomial identities.

(For an application of the same trick to Lie algebras, see [32], discussion following Proposition 4.1, and also $\$ 9$ thereof.)

We end this section by examining the behavior of universal right, left and two-sided inversion under some special hypotheses on the map $f: P \rightarrow Q$ of finitely generated projective $R$-modules to be inverted.

Suppose that $f$ is already left invertible. This means that up to isomorphism $f$ is the inclusion of the summand $P$ in a direct sum $Q=P \oplus Q^{\prime}$; a left inverse will be the projection $j: P \oplus Q^{\prime} \rightarrow P$. Now suppose that we universally adjoin to $R$ another left inverse, getting $S=R\left\langle g: \bar{Q} \rightarrow \bar{P} ; g \bar{f}=1_{\bar{P}}\right\rangle$. Then $g: \bar{Q}=\bar{P} \oplus \bar{Q}^{\prime} \rightarrow \bar{P}$ must have the form $\left(1_{\bar{P}}, h\right)$ where $h$ is a map of $\bar{Q}^{\prime}$ to $\bar{P}$, and in fact, it is easy to see that $S$ will be isomorphic to the universal mapping ring $\left.R \backslash h: \bar{Q}^{\prime} \rightarrow \bar{P}\right\rangle$, which has all desirable properties, by Theorem 5.3.

Suppose, on the other hand, that we universally adjoin a right inverse to this left-invertible map $f$; equivalently, that we adjoin a 2-sided inverse; equivalently, that we set $\overline{f j}=1_{\bar{Q}}$ (where $j$ is a left inverse to $f$, as above). Note that $1-f j: Q \rightarrow Q$ is the projection onto the summand $Q^{\prime}$; we conclude that $R\left\langle\bar{f}^{-1}\right\rangle=R\left\langle\bar{Q}^{\prime}=0\right\rangle=R / T_{Q^{\prime}}\left(T_{Q^{\prime}}=\right.$ the trace ideal of $Q^{\prime}$. For definition and basic properties of $T_{Q^{\prime}}$ cf. [4, Proposition 3.4].) Conversely, every case of the construction $R / T_{P}$ can be described as such an inversion.

One more case: suppose $f: P \rightarrow P$ is an idempotent endomorphism of a single module $P$, inducing a decomposition $P=P^{\prime} \oplus P^{\prime \prime}$. Then it is easily seen that to right, left, or two-sidedly invert $f$ all come to the same construction: $R\left\langle P^{\prime \prime}=0\right\rangle=R / T_{P^{\prime \prime}}$.

(A common generalization of a 1-sided invertible map and an idempotent map is a map $f: P \rightarrow Q$ having a quasi-inverse-a $j: Q \rightarrow P$ such that $f j f=$ $f$ and $j f j=j .\left({ }^{7}\right)$ The above observations can be generalized to this case. Con-

(7) Such an $f$ is sometimes called a regular map (cf. [26, p. 21, Exercise 7], and the term "(von Neumann) regular ring"). Unfortunately the terms quasi-invertible and regular both have other conflicting uses. 
ceivably, the adjunction of universal quasi-inverses might also show interesting properties.)

It follows that any general results holding for the construction $R\left\langle\bar{f}^{-1}\right\rangle$ without restriction on $f$ will also hold for the construction $R\langle\bar{P}=0\rangle$.

11. The construction $R\langle\bar{P}=0\rangle$. Aside from being a special case of localization, note that this is also the case of the construction $R\left\langle i, i^{-1}: \bar{P} \cong \bar{Q}\right\rangle$ where $Q$ is the zero module. (We were able to get good results for this construction only assuming $P$ and $Q$ both nonzero.)

The trace ideal of a finitely generated projective $R$-module $P$ is finitely generated, and idempotent: $T_{P} T_{P}=T_{P}$. Hence when $R$ is commutative, it is generated by an idempotent element of $R$. (The characteristic function of the open-closed subscheme of $\operatorname{Spec} R$ where rank $P>0$.) Thus given $P$ we get a decomposition $R=S \times T$ as rings, and the map $R \rightarrow R / T_{P}=R\langle\bar{P}=0\rangle$ is just the projection of $R$ onto the factor $S$. This map has all the good properties one could hope for: Clearly, $\operatorname{Mod} R \approx \operatorname{Mod} S \times \operatorname{Mod} T ; S$ is projective over $R$, and every $S$-module $N$ can be lifted to an $R$-module $M$ of the same homological dimension.

Many of these properties are lost in the noncommutative case. Of course, since $S=R / T_{P}$ is a homomorphic image of $R$, one can lift all $S$-modules to $R$-modules; but one cannot, in general, preserve their homological dimensions: [6, §III] gives an example in which $R / T_{\boldsymbol{P}}$ has finitely generated projective modules not liftable to finitely generated projective $R$-modules. However, I know of no such example where $R$ is hereditary! Also, $R / T_{P}$ need not be flat over $R$ : Let $R=k\left\langle x, y ; y^{2}=y\right\rangle$, which is hereditary by the results of $\S 5$, and let $P$ be the image of the idempotent map $1-y: R \rightarrow R$. Then $T_{P}$ is the ideal generated by $1-y$, and $R / T_{P} \cong k[x]$, via $y \mapsto 1$. Now $R$ has for $k$-basis the set of words in $\{x, y\}$ having no subwords $y y$. It is easy to deduce from this and the defining relation for $R$ that the equation $a \cdot x=b \cdot x y$ has no nonzero solution in $R$. Yet in $R / T_{P}$ it has the solution $a=b=1$, proving nonflatness as in the preceding section.

Again, we give as test-problems the questions of comparing homological and global dimensions over $R$ and $S$.

The technique of $\S 5$ gives, with no special trickery needed this time:

LEMMA 11.1. If one of the following statements is true, all are:

(i) If $R$ is a k-algebra and $P$ any finitely generated projective $R$-module, then $\mathrm{r} \mathrm{gl} \operatorname{dim} R\langle\bar{P}=0\rangle \leqslant \mathrm{r} g l \operatorname{dim} R$.

(ii) If $R$ is a k-algebra and $e$ any idempotent element of $R$, then $\mathrm{r} \mathrm{gl} \operatorname{dim} R / R e R \leqslant \mathrm{r} \mathrm{gl} \operatorname{dim} R$. 
(iii) Let $k^{\prime}$ denote the field $k$, made a $(k \times k)$-ring via the second projection map, $k \times k \rightarrow k$. If $R$ is $a(k \times k)$-ring $k_{k}$, then $\mathrm{r} g l \operatorname{dim} R \Perp_{k \times k} k^{\prime}$ $\leqslant \mathrm{rgl} \operatorname{dim} R$.

Here (i) and hence also (ii), (iii) are true when $\mathrm{r} g l \operatorname{dim} R \leqslant 1$, by results of Eilenberg, Nakayama and Nagao on $\mathrm{r} g l \operatorname{dim} R / I$, where $R$ is any hereditary ring and $I$ any eventually idempotent ideal [19] (generalized by Fields [20]).

Note that if $R$ is a $k$-algebra, the constructions $R\langle\bar{P}=0\rangle$ and $R\left\langle\bar{f}^{-1}\right\rangle$ (but not the one-sided inverse constructions) will give the same results whether performed in the category of $k$-algebras or of rings. (This is basically because they yield epimorphisms $R \rightarrow S$, and if $x$ is central in a ring $R, \bar{x}$ will be central in any epimorph $S$ of $R$ [29].) I would guess that these two constructions will behave as well or badly for arbitrary rings, i.e., Z-algebras $R$, as for algebras over a field.

12. A unifying viewpoint, and ideas for a generalization. In this section, if $R$ is a ring $M d l R$ will denote the category of all right $R$-modules (not necessarily finitely generated). The commutative ring $k$ will not be assumed a field except when we so specify, or when we refer to the constructions of preceding sections.

Note that the construction considered in $\S 7$ was a particularly simple case of the general construction $S=R\langle B\rangle$ : The $k$-linear category $B$ we used had only a single object $X$, and was thus determined by the $k$-algebra $\operatorname{Hom}_{B}(X, X)$, which we called $B$. The $k$-linear category $A$ was given the minimum compatible structure: one object (call it $X$ also) with $\operatorname{Hom}_{A}(X, X)=k$. Writing $F(X)=$ $P \in P-M o d R$, we got a ring $S=R\langle B\rangle=R\langle B \rightarrow$ End $\bar{P}\rangle$, whose homological properties were closely linked to those of $R$ and $B$. In particular, for $R=k$ and $P=k^{n}$ the result was a ring $\mathfrak{w}_{n}(B)$ whose module theory was close to that of $B$.

To try to generalize these results to $k$-linear categories $B$ with more than one object, we want to associate to such a $B$ a "module theory", as in the oneobject case. Now it is a familiar observation that (right or left) modules over a $k$-algebra $B$ can be described as (covariant or contravariant) additive functors from $B$, as a one-object category, into the category $A b$ of abelian groups; equivalently, as (covariant or contravariant) $k$-linear functors of this category into $M d l k$. So given any small $k$-linear category $B$, let us define $M d l B$ (respectively $M d l B^{(l)}$ ) to be the $k$-linear category of linear covariant (respectively contravariant) functors $M: B \rightarrow A b$; equivalently, of $k$-linear functors $M$ : $B \rightarrow M d l k$.

For an extensive development of this idea and its consequences, the reader 
is referred to Mitchell [28]. In the next few paragraphs, I shall mention some key points that will be of importance to us.

When the category $B$ has only finitely many objects, one can in fact construct from it a $k$-algebra $B$ such that $M d \ell B$ is equivalent as $k$-linear category to $M d l B$ [28, Theorem 7.1]. Namely, take $B$ to consist of all formal $\mathrm{Ob}(B) \times \mathrm{Ob}(B)$ matrices, with a member of $\operatorname{Hom}_{B}(Y, X)$ in the $(X, Y)$ position for each $(X, Y) \in \mathrm{Ob}(B) \times \mathrm{Ob}(B)$. To any $M \in M d l B$ we now associate $\bigoplus_{\mathrm{Ob}(B)} M(X)$, made a $B$-module in the obvious fashion (matrices acting on rows!); the argument showing this is an equivalence of categories is straightforward.

This equivalence can also be deduced from two general observations. Suppose $B$ is a full subcategory of $B^{\prime}, k$-linear categories with arbitrarily many objects, and that either (i) every object of $B^{\prime}$ is a direct sum of objects in the subcategory $B$ (i.e., its identity map is a sum of orthogonal idempotents $j i$ such that $i j=1_{X}$ with $X \in \mathrm{Ob}(B)$ ) or that (ii) every object of $B^{\prime}$ is a direct summand (retract) of an object of the subcategory $B$. In either case the restriction functor $M d l B^{\prime} \rightarrow M d l B$ is easily seen to be an equivalence. Now if $B$ is a $k$-linear category with only finitely many objects, we can throw in one new object $W$, behaving as a direct sum of the rest, then delete all the old objects since they are retracts of $W$. In the resulting one-object category, which has the same module-theory as $B$, the associated ring $\operatorname{Hom}(W, W)$ is the " $B$ " just described.

(This trick can also be used to get from a one-object category $B_{1}$ to another one-object category $B_{2}$ whenever the associated $k$-algebras $B_{1}$ and $B_{2}$ are Morita equivalent! Namely, if $B_{2}=$ End ${ }_{B_{1}} P$, where $P$ is a direct summand in $B_{1}^{n}$, and $B_{1}$ a direct summand in $P^{m}$, consider the chain of full subcategories of $\operatorname{Mod} B_{1}$, with objects:

$$
\left\{B_{1}\right\} \subseteq\left\{B_{1}, B_{1}^{n}\right\} \subseteq\left\{B_{1}, B_{1}^{n}, P\right\} \supseteq\left\{B_{1}, P\right\} \subseteq\left\{B_{1}, P^{m}, P\right\} \supseteq\left\{P^{m}, P\right\} \supseteq\{P\}
$$

The rings associated with the first and last step are $B_{1}$ and $B_{2}$ respectively.)

(One can also "embed" the structure of any $k$-linear category $B$ in a $k$ algebra $B$, by using infinite matrices differing at only finitely many places from "scalar" matrices. Then $M d l B$ turns out to be equivalent to a certain full subcategory of $M d l B$. Thus the homological properties of $B$ do not coincide with those of $B$, though they can presumably be studied with the help of the latter.)

Now let $k$ be a field and $B$ a $k$-linear category with $r<\infty$ objects, all nonzero. Let $A$ be the category with the same objects as $B$, but with $\operatorname{Hom}_{A}(X, Y)$ defined to be $k$ if $X=Y, 0$ otherwise. Let $G: A \rightarrow B$ be 
the unique $k$-linear functor which is the identity on objects. For each $X \in$ $\mathrm{Ob}(\mathrm{A})$, choose any nonzero object $F(X)$ in $\mathrm{Ob}(\mathrm{P}-\mathrm{Mod} k)$ and let $F: A \rightarrow$ $P$-Mod $k$ be the unique $k$-linear functor taking each $X$ to $F(X)$. By Theorem 3.3 we have a $k$-algebra $S=k\langle B\rangle$ universal for the diagram:

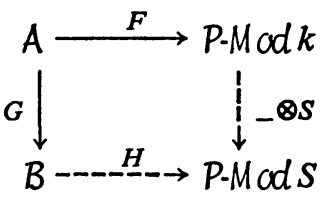

We can now show how to generalize the method of $\S 7$ and study the moduletheory of $S$ in terms of the module-theory of $B$. As a first step, we replace $A$ and $B$ by one-object categories $A^{\prime}$ and $B^{\prime}$ having the same module-theories, as described above, and call the endomorphism rings of their unique objects $A$ $=r^{k}$ and $B$. The functor $G: A \rightarrow B$ induces a functor $G^{\prime}: A^{\prime} \rightarrow B^{\prime}$, i.e., a homomorphism $A \rightarrow B$ of $k$-algebras. The functor $F: A \rightarrow$ P-Mod $k$ induces a functor $F^{\prime}: A^{\prime} \rightarrow$ P-Mod $k$ taking the unique object of $A^{\prime}$ to $k^{n} \in$ P-Mod $k$, where $n=\Sigma_{\mathrm{Ob}(\mathrm{A})} \operatorname{dim}_{k} F(X)$; i.e., a $k$-algebra homomorphism $A \cong$ $k^{r} \rightarrow \mathfrak{m}_{n}(k)$. One now finds that $\left.k\langle B\rangle \cong k\left\langle B^{\prime}\right\rangle \cong \mathfrak{M}\right\rangle_{n}^{-1}\left(B \Perp_{A} \mathfrak{m}_{n}(k)\right)$. (This is not a case of the construction $\mathfrak{w}_{n}$ except when $A$ has only one object, so that $A=k$.) Hence using the theory of coproducts [7] as in preceding sections, one can relate the theory of $k\langle B\rangle$-modules to that of $B$-modules; for example $\mathrm{r} \mathrm{gl} \operatorname{dim} k\langle B\rangle \leqslant \max (1, \mathrm{r} \mathrm{gl} \operatorname{dim} B)$.

Suppose we generalize the above construction by letting $F$ map $A$ into P-Mod $R$ for some $k$-algebra $R$, rather than into P-Mod $k$. A complication arises: $\bigoplus F(X)$ may not be a free $R$-module $R^{n}$. To cure this, we choose a nonzero $U \in P$-Mod $R$ which, when added to the above sum, makes it free. (Sound familiar?) Then add to each of $A$ and $B$ one additional object, $U_{0}$, with endomorphism-algebra $k$ in both categories, and otherwise only zero homomorphisms in and out. Extend $F$ to send the new object of $A$ to $U$. The universal $k$-algebra for the extended diagram will be the same as for the old one, since we are adjoining no new maps or relations. We can now complete the construction as above. This construction includes all those studied in $\S 5$ as well as $\S 7$ ! The algebras $k \times k \times k, \mathfrak{m}_{2}(k) \times k, \mathfrak{t}_{2}(k)$ $\times k$ etc. used as tools in $\S 5$ are now seen to be the algebras $A$ and $B$ associated with appropriate categories $A$ and $B$.

Consider finally the situation where $B$ consists of a family of objects $X_{1}, \cdots, X_{r}$, with no homomorphisms between distinct objects, so that $B$ is determined by the endomorphism algebras $B_{i}=\operatorname{Hom}_{B}\left(X_{i}, X_{i}\right)$. Let $A$, as above, have the same objects as $B$ but minimal structure of $k$-linear category; and map $A$ into P-Mod $k$ by sending all objects to the free module of rank 1 . One finds that the universal $k$-algebra $k\langle B\rangle$ is then the coproduct $B_{1} \Perp_{k} \cdots$ 
$\Perp_{k} B_{n}$. Note that a $B$-module corresponds to an $n$-tuple $\left(M_{i}\right)$ of modules over the $B_{i}$, which becomes one module $M_{1} \times \cdots \times M_{n}$ over the "Morita equivalent" ring $B_{1} \times \cdots \times B_{n}$, which induces a "standard module" $\bigoplus M_{i} \otimes S$ over $S$. The formula for the global dimension of a coproduct [7, Corollary 2.5] is seen to be related to the fact that the global dimension of a $k$-linear category $B$ (i.e., $\max h \operatorname{dim} M, M \in M d l B$ ) is the supremum of the dimensions of its connected components; and similarly for other results.

It is now time to ask, what about categories $B$ with infinitely many objects? For instance, suppose $B$ is any small $k$-linear category, let $G: A \rightarrow B$ be constructed as before, let $F: A \rightarrow P$-Mod $k$ be any faithful $k$-linear functor, and $S=k\langle B\rangle$ as in Theorem 3.3. Given any $B$-module $M: B \rightarrow M d l k$, we can define an $S$-module $\bar{M}$. (The idea is: just as for a ring homomorphism $R \rightarrow S$, the restriction-of-scalars functor $M d l S \rightarrow M d l R$ has a left adjoint $\_\otimes S$ : $M d l R \rightarrow M d l S$, so for a functor among small $k$-linear categories, $F: B \rightarrow$ $B^{\prime}$, the functor composition-with- $F$, taking $M d l B^{\prime}$ to $M d l B$ has a left adjoint $-\otimes_{B} B^{\prime}$. One also needs the observation that $\operatorname{Mdl}(P-\operatorname{Mod} S)$ is equivalent to $M d l(S)$.) If we call the $S$-modules that so arise "fundamental", can we again show that every submodule of a fundamental $S$-module is isomorphic to a fundamental $S$-module? describe $S_{\oplus}($ Fund $S)$ in terms of $S_{\oplus}(\operatorname{Mod} B)$ ? bound $\mathrm{r} g l \operatorname{dim} S$ by $\max (\mathrm{r} \mathrm{gl} \operatorname{dim} B, 1)$ ?

In the special case where $B$ is totally disconnected and $F$ takes all objects of $A$ to the free $k$-module of rank 1, the answer is yes, by the results of [7]. (We can even replace the former hypothesis by "the connected components of $B$ have finitely many objects", and drop the latter, by appropriate arguments.)

I believe that in fact such results are true for $B$ and $F$ arbitrary, but that they cannot be obtained from the results of [7] as given, but rather, by further extension of the method of that paper. In particular, the process of obtaining canonical forms for elements of $S$ and $\bar{M}$ would mix the ideas of [7, §4] and the proof of Theorem 8.1 of this paper. I can see no obstruction to carrying out the whole analysis of [7] when $F$ takes all objects of $A$ to $k$-modules of dimension 1 . The details in the general case are less clear.

This generalization is still not as good as we would like, since taking $B$ totally disconnected, we get a general coproduct of $k$-algebras over a field $k$, while results of [7] apply to coproducts of $R_{0}$-rings over any ring $R_{0}$ of global dimension zero. I shall sketch below some ideas of what I think the proper generalization should be like, but not try to formulate it precisely.

First, let us free the construction of $k$. Following [28] we understand an additive category to mean a Z-linear category (called by some a preadditive category, in [26] an Ab-category.)

In place of the ring $R_{0}$ of global dimension 0 of [7], we should take 
$A$ to be an additive category of zero global dimension (meaning, as for rings, the global dimension of its module category $M d l A$ ). It is not hard to show for an additive category $A$ that $r \operatorname{gl} \operatorname{dim} A=0$ if and only if $A$ can be reduced via the method of putting in and throwing out direct sums and direct summands ("Morita equivalence of linear categories") to a disjoint union $A^{\prime}$ of one-object categories whose endomorphism rings are skew fields (possibly infinitely many, in constrast to the corresponding result of ordinary ring theory!). Further, if $F$ : $A \rightarrow B$ is an additive functor, these changes in $A$ can be "paralleled" in $B$, giving a functor $F^{\prime}: A^{\prime} \rightarrow B^{\prime}$; so without loss of generality, we should be able to restrict attention to the case where $A$ is a category of just that sort.

What should be the result of the construction? Up to now we have been constructing rings $S$. But if it is desirable to generalize our hypotheses to allow additive categories in place of rings, we should likewise generalize the construction to yield an additive category which may have more than one object.

Let us observe, as an example, how the coproduct over a skew field $R_{0}$ of a family $\left(R_{\lambda}\right)_{\Lambda}$ of $R_{0}$-rings can be described as a pure additive category construction. The four categories we shall set up will each have for object-set the given index-set $\Lambda$. Define $A$ by putting $\operatorname{Hom}_{A}\left(\lambda, \lambda^{\prime}\right)=R_{0}$ if $\lambda=\lambda^{\prime}, 0$ otherwise. Define $B$ by $\operatorname{Hom}_{B}(\lambda, \lambda)=R_{\lambda}$, with all other Hom-sets again 0 . In $C$, take $\operatorname{Hom}_{C}\left(\lambda, \lambda^{\prime}\right) \doteq R_{0}$ for all $\lambda$ and $\lambda^{\prime}$, with composition given by multiplication in $R_{0}$-in effect, $C$ is $A$ with all objects made isomorphic in a coherent fashion. We now form the pushout among categories with object-set $\Lambda$ :

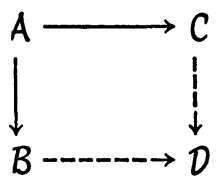

In $D$, as in $C$, all objects are isomorphic, and the endomorphism ring of any one of them will be the desired coproduct $\amalg R_{\lambda}$. Alternatively, instead of making all categories have the same objects, we might have made all objects of A fall together into one object in $C$, and hence $D$, and gotten $\amalg_{\lambda}$ as the endomorphism ring of the unique object of $D$.

One might ask whether to be a generalization of the construction we have been considering, the above diagram should not have P-Mod $C$ and P-Mod D where we show $C$ and $D$. Actually, for $R$ a ring, $P-M o d R$ is, in a sense that can be made precise, the largest additive category "Morita equivalent to" the one-object category determined by $R$. We did not really need such a large category in our constructions; we could with a little more wordage have formulated the results of $\S 3$ with $P$-Mod $R$ and P-Mod $S$ replaced by an arbitrary full subcategory of the former, and the induced subcategory of the latter-but to no 
particular advantage. Now for $C$ a general additive category one can similarly define $\mathrm{P}$-Mod $\mathrm{C}$-it will consist of all formal direct summands of all formal direct sums of objects of $C$, and have the same maximality property-but since $C$ was perfectly general anyway, there is now no added convenience in this move.

The schema suggested has one unsatisfying feature: there seems to be a redundancy in that "amalgamation" comes in two ways-putting together the categories $B$ and $C$, which now play equivalent roles, and putting together the "ring" structures in each of them. Would a better formulation be a difference-cokernel construction, $A \rightrightarrows B \longrightarrow D$ ? (Cf. the 'generalized free product groups' of [33].) Perhaps. One must aim for results that maximize formal and conceptual simplicity yet are applicable to as wide a range of cases as possible. Sometimes this can be done by an ingenious reduction to a simple case, other times, compromise is required. Correct solutions are not unique.

I would be interested in hearing from anyone who does pursue these lines of investigation!

\section{Appendix: Why finitely generated projective modules? If $P$ and $Q$} are modules over a $k$-algebra $R$, it is clear that to study the properties of universal constructions such as $S=R\left\langle i, i^{-1}: \bar{P} \cong \bar{Q}\right\rangle$ by the methods used here (matrix reduction to the case $P=e_{P} R$, so that $S$ becomes a coproduct ring over a direct product of copies of $k), P$ and $Q$ must be assumed finitely generated and projective. What is not as obvious is the need for such hypotheses in the theorems of $\$ 3$ which establish the existence of these universal rings.

Suppose we try to generalize the construction of Theorem 3.1 as follows. Let $M$ and $N$ be modules over a $k$-algebra $R$, with $N$ finitely presented. Write down presentations for both modules:

$$
\begin{aligned}
& F_{1} \stackrel{a}{\longrightarrow} F_{2} \longrightarrow M \longrightarrow 0, \\
& F_{3} \longrightarrow{ }^{b} F_{4} \longrightarrow 0
\end{aligned}
$$

where all $F_{i}$ are free, and $F_{3}, F_{4}$ of finite rank. Note that for any $R$-ring $T$, we have presentations of $T$-modules:

$$
\begin{aligned}
& F_{1} \otimes T \stackrel{\bar{a}}{\longrightarrow} F_{2} \otimes T \longrightarrow M \otimes T \longrightarrow 0, \\
& F_{3} \otimes T \longrightarrow \stackrel{\bar{b}}{\longrightarrow} F_{4} \otimes T \longrightarrow N \otimes T \longrightarrow 0 .
\end{aligned}
$$

Using Theorems 3.1 and 3.2, we can construct an extension ring $S=$ $\left.R \backslash d: F_{1} \rightarrow F_{3}, e: F_{2} \rightarrow F_{4} ; \bar{b} d=e \bar{a}\right\rangle:$ 


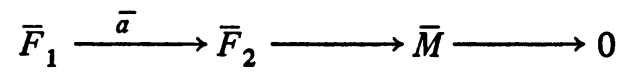

(10)

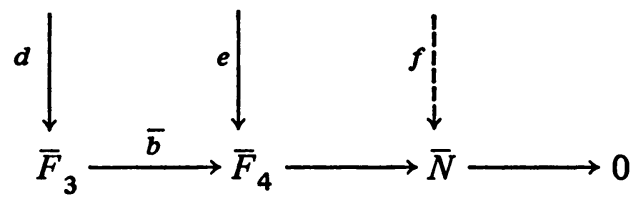

These $d$ and $e$ induce a map $f: \bar{M} \rightarrow \bar{N}$. However, $S$ and $f$ will not have the universal property of Theorem 3.1. Rather, it will have the property that given any $R$-ring ${ }_{k}, T$, any homomorphism of $T$-modules $g: M \otimes T \rightarrow N \otimes T$, and a specified lifting of $g$ to a map of presentations (9), there exists a unique homomorphism $S \rightarrow T$ under which (10) induces this map of presentations. In particular, $S$ has the weak universal property that given $T$ and $g$ as above, there will exist a not necessarily unique homomorphism $S \rightarrow T$ relative to which $g=f \otimes T$.

If we take for $M$ the free $R$-module of rank 1 , then a universal or weakly universal map $M \otimes_{-} \rightarrow N \otimes_{-}$simply means a universal or weakly universal choice of element of $N \otimes_{-}$. In this case, we do not even need $N$ finitely related to get such a weakly universal element:

Proposition 13.1. Let $k$ be a commutative ring, $R$ a $k$-algebra, and $N$ an $R$-module. Then the following conditions are equivalent:

(i) $N$ is finitely generated.

(ii) There exists an $R$-ring $g_{k} S$, and an element $x \in N \otimes S$, such that for any $R$ :ring ${ }_{k} T$ and any element $y \in N \otimes T$, there exists $a$ (not necessarily unique) homomorphism of $R$-rings, $S \rightarrow T$, with respect to which $y=\bar{x} \in$ $N \otimes T$.

Proof. (i) $\Rightarrow$ (ii) Note that for modules $M$ and $N$ as discussed above, if we put $S=R\left\langle g: \bar{M} \rightarrow \bar{F}_{4}\right\rangle$, then the composed map $\bar{M} \stackrel{g}{\rightarrow} \bar{F}_{4} \rightarrow \bar{N}$ will have the weak universal property if $M$ is a projective $R$-module, by the maplifting definition of projectivity. So this is true in particular when $M$ is free of rank 1 .

(ii) $\Rightarrow$ (i) The element $x \in N \otimes S$ will lie in a submodule induced by some finitely generated submodule $N_{0} \subseteq N$. Under any homomorphism of $R$ rings, $S \rightarrow R$, we see that $x$ will be taken to an element of $N_{0}$. Applying the weak universal property with $T=R$, we conclude that $N=N_{0}$, so $N$ is finitely generated.

The next theorem will show that the condition for the existence of true universal maps is more stringent. We first need:

Lemma 13.2. Let $k$ be a field, $R$ a k-algebra, and $N$ an $R$-module. 
Then the following conditions are equivalent:

(i) $N$ is flat; i.e., for every one-to-one map $L \rightarrow L^{\prime}$ of left $R$-modules, the map of abelian groups $N \otimes L \rightarrow N \otimes L^{\prime}$ is one-to-one.

(ii) For every one-to-one map $B \rightarrow B^{\prime}$ of $R$-bimodules , $_{k}$, the map of right $R$-modules $N \otimes B \rightarrow N \otimes B^{\prime}$ is one-to-one.

(iii) For every one-to-one map $T \rightarrow T^{\prime}$ of $R$-rings ${ }_{k}$, the induced map of extended modules $N \otimes T \rightarrow N \otimes T^{\prime}$ is one-to-one.

Proof. (i) $\Rightarrow$ (ii) $\Rightarrow$ (iii) is clear. To see (i) $\Leftarrow$ (ii) $\Leftarrow$ (iii), note that if $L \rightarrow L^{\prime}$ is a one-to-one map of left $R$-modules, then the map of $R$-bimodules, $L \otimes_{k} R \rightarrow L^{\prime} \otimes_{k} R$ is, as a map of left modules, a nonvacuous "direct sum of copies" of the given map since $R$ is free as a $k$-module, and that condition (ii) applied to this map gives (i) for the first map. Likewise, if $B \rightarrow B^{\prime}$ is as in (ii), then one can get a map of $R$-rings ${ }_{k}, R \oplus B \rightarrow R \oplus B^{\prime}$ (B and $B^{\prime}$ being given zero internal multiplication), and applying (iii) to this map gives (ii) for the map of $B$ 's.

(The assumption that $k$ was a field was used to see that $R$ was free as a $k$-module, so that $L \otimes_{k} R \rightarrow L^{\prime} \otimes_{k} R$ would have the desired relation to $L \rightarrow L^{\prime}$. There are many other conditions under which left $R$-modules can be embedded as summands in bimodules, e.g., if $R$ is commutative. It would be nice if one could prove Lemma 13.2 without restriction on $k$ ).

We can now prove:

THEOREM 13.3. Let $k$ be a field, $R$ a k-algebra, and $N$ an $R$-module. Then the following conditions are equivalent:

(i) $N$ is finitely generated and projective as an $R$-module.

(ii) For every $R$-module $M$, there exists an $R$-ring ${ }_{k} S=R\langle f: \bar{M} \rightarrow \bar{N}\rangle$ with a universal map $f: \bar{M} \rightarrow \bar{N}$ (as in Theorem 3.1. More generally, for every R-ring $_{k}, T$, and T-module $M$, there exists a T-ring $\left.\left.{ }_{k}, T\right\}: \bar{M} \rightarrow \bar{N}\right\rangle$ with the corresponding universal property over $T$ ).

(iii) There exists an $R$-ring ${ }_{k} S=R\langle x \in \bar{N}\rangle$ with an element $x \in N \otimes S$ such that for any $R$-ring ${ }_{k} T$, and any element $y \in N \otimes T$, there exists $a$ unique homomorphism of $R$-rings $S \rightarrow T$ with respect to which $y=\bar{x}$.

Proof. (i) $\Rightarrow$ (ii) by Proposition 3.1, and (ii) $\Rightarrow$ (iii) by taking $M=R$.

Assuming (iii) we see by Lemma 13.1 that $N$ is finitely generated. We claim that it is in fact finitely presented. Let $F_{3} \rightarrow F_{4} \rightarrow N \rightarrow 0$ be a free presentation of $N$, with $F_{4}$ finitely generated. Construct by Theorem 3.1, with $R$ for $M$ and $F_{4}$ for $P$, a ring $R^{\prime}=R\left\langle z \in \bar{F}_{4}\right\rangle$, and let $y$ denote the image in $N \otimes R^{\prime}$ of $z \in F_{4} \otimes R^{\prime}$. Now taking $S$ as in (iii), one can construct, first, a unique map $S \rightarrow R^{\prime}$ with respect to which $\bar{x}=y \in N \otimes R^{\prime}$, 
and secondly, a unique map $S \rightarrow R^{\prime}$ with respect to which $\bar{x}=0 \in N \otimes R^{\prime}$. Form their difference-cokernel: $S \rightrightarrows R^{\prime}-\rightarrow U$ Then it is immediate that the image of $z \in F_{4} \otimes R^{\prime}$ will be an element $w \in F_{4} \otimes U$ which goes to zero in $N \otimes U$, and that the pair $U, w$ is universal for this property. From our presentation of $N$, we see that $w$ will lie in the image of $F_{3} \otimes U$. Hence it will in fact lie in the image of $F_{3} \otimes U$, where $F_{3}^{\prime} \subseteq F_{3}$ is the submodule spanned by a finite subset of the basis. It is easy to deduce from the universal property of $w$ that $F_{3}^{\prime} \rightarrow F_{4} \rightarrow N$ is a finite presentation of $N$, as desired.

Finally, note that if $T \rightarrow T$ is a homomorphism of $R$-rings ${ }_{k}$, then the induced module map $N \otimes_{R} T \rightarrow N \otimes_{R} T^{\prime}$ can, by the universal property of $S$, be identified with the induced set map

$$
\operatorname{Hom}_{R-\operatorname{ring}}(S, T) \rightarrow \operatorname{Hom}_{R-\operatorname{ring}}\left(S, T^{\prime}\right)
$$

If $T \rightarrow T^{\prime}$ is one-to-one, so will this map be, hence so will the module-map. Hence by Lemma $13.2, N$ is a flat $R$-module.

A flat finitely presented module is projective, so $N$ is finitely generated and projective, establishing (i).

In particular, the equivalence of (i) and (iii) can be restated: The functor from $R$-rings $k_{k}$ to sets, $T \mapsto N \otimes T$, is representable (cf. [20], [26]) if and only if the $R$-module $N$ is finitely generated and projective.

Note that we had to assume $k$ a field only to apply Lemma 13.2.

The above result does not exclude the possibility that for some special classes of $M, R\langle f: \bar{M} \rightarrow \bar{N}\rangle$ may exist for other than finitely generated projective $N$. (There is the trivial case $M=0, N$ arbitrary.)

Despite Theorem 13.3, there is a different way in which the $N$ of the construction $R\left\langle f: M \otimes_{-} \rightarrow N \otimes_{-}\right\rangle$can be generalized: the operation $N \otimes_{-}$ can be replaced by a more general sort of functor from $R$-rings , $_{k} T$, to $T$ modules $N(T)$. E.g., let $X$ be an infinite set; then the extension of $R$ by an $X$-tuple of indeterminates can clearly be described as $R\left\langle\bar{R} \rightarrow \bar{R}^{X}\right\rangle$, i.e., it is an $R$-ring ${ }_{k} S$ with a universal element of the power module $S^{X}$. Likewise, for $a \in R$, the ring $R\langle x ; \bar{a} x=0\rangle$ has the universal property of $R\left\langle\bar{R} \rightarrow \mathrm{rt} \operatorname{ann}_{\bar{R}}(\bar{a})\right\rangle$.

The subject is a tricky one. For instance, from what we have just said, $T \mapsto \operatorname{Hom}\left(T, T^{X}\right)$ and $T \mapsto \operatorname{Hom}\left(T, \mathrm{rt} \operatorname{ann}_{T} \bar{a}\right)$ are representable functors, though $T \mapsto \operatorname{Hom}\left(T,\left(R^{X}\right) \otimes T\right)$ and $T \mapsto \operatorname{Hom}\left(T,\left(\mathrm{rt} \mathrm{ann}_{R} a\right) \otimes T\right)$ are generally nonrepresentable functors. On the other hand, one finds that $T \mapsto$ $\operatorname{Hom}\left(T^{X}, T\right)$ and $T \mapsto \operatorname{Hom}\left(\mathrm{rt} \mathrm{ann}_{T} \bar{a}, T\right)$ are not functors at all!

Let me outline one general positive result.

Functors of the form $M \otimes$ _ can be described as associating to an $R$-ring $T$ the cokernel $\bar{M}$ of the map: 


$$
\bigoplus_{A} a T \stackrel{\bar{c}}{\longrightarrow} \bigoplus_{B} b T-\cdots \bar{M}
$$

where $c$ is a column-finite $B \times A$ matrix over $R$, and $\bigoplus a R \stackrel{c}{\rightarrow} \bigoplus b R \rightarrow M$ is a presentation of the $R$-module $M$. Now dually, consider a functor associating to an $R$-ring $T$ the kernel $K(T)$ of the map:

$$
K(T) \stackrel{-\cdots}{\rightarrow} T^{X} \stackrel{\bar{z}}{\longrightarrow} T^{Y}
$$

where $z$ is a row-finite $Y \times X$ matrix over $R$. (The two functors we just introduced are of this form.) These can be looked at another way. A general left $R$-module $L$ will be given by the cokernel of the map:

$$
L \leftarrow---\bigoplus_{X} R x \stackrel{z}{\longleftarrow} \bigoplus_{Y} R y,
$$

with $z$ as in (12). The kernel $K(T)$ of (12) can now be described as $\bar{L}^{*}$, where $\bar{L}$ denotes the left $T$-module $T \otimes L$, and $*$ denotes the duality functor $\operatorname{Hom}(-, T)$, taking left $T$-modules to right $T$-modules. One can now show:

Proposition 13.4. Let $k$ be a commutative ring, $R$ a k-algebra, $M$ any right $R$-module, and $L$ any left $R$-module. Then the following universal rings exist, and may be identified with one another. (I.e., the functors $R$-ring $g_{k} \rightarrow$ Set that they represent are isomorphic.)

(i) $S=R\left\langle f: \bar{M} \rightarrow \bar{L}^{*}\right\rangle$, an $R$-ring ${ }_{k}$ with a universal map of right $S$ modules, $M \otimes S \rightarrow(S \otimes L)^{*}$.

(i*) $S=R\left\langle f^{*}: \bar{L} \rightarrow \bar{M}^{*}\right\rangle$, an $R$-ring ${ }_{k}$ with a universal map of left $S$ modules, $S \otimes L \rightarrow(M \otimes S)^{*}$.

(ii) $S=R\left\langle g \in\left(\bar{L} \otimes_{k} \bar{M}\right)^{*}\right\rangle$, where “*” now means $\operatorname{Hom}_{(S, S) \cdot B \text { imod }}(-, S)$.

(ii') $S=R\left\langle L \otimes_{k} M\right\rangle$, the tensor-ring over $R$ on the $(R, R)$-bimodule $B=L \otimes_{k} M$. (Explicitly: $\left.S=R \oplus B \oplus \cdots \oplus\left(B \otimes_{R} \cdots \otimes_{R} B\right) \oplus \cdots\right)$

A special (and obvious) case of the existence-assertion of the above theorem is the observation that a kernel-functor as in (12) is always representable. Combining this with the restriction given by Theorem 13.3 on when a cokernel-functor as in (11) is representable, we get conditions for when a kernel-functor and a cokernel-functor can be isomorphic, i.e., when a certain kind of sequence can be functorially exact:

Corollary 13.5. Let $k$ be a field, $R$ a k-algebra, $X, Y, A, B$ sets, $c$ a column-finite $B \times A$ matrix, $z$ a row-finite $Y \times X$ matrix, and $m$ an arbitrary $A \times Y$ matrix over $R$. Suppose that for all $R$-rings, $k_{k}, T$, the sequence of right T-modules: 


$$
\bigoplus_{A} a T \stackrel{\bar{c}}{\longrightarrow} \bigoplus_{B} b T \stackrel{\bar{m}}{\longrightarrow} T^{X} \stackrel{\bar{z}}{\longrightarrow} T^{Y}
$$

is exact. Then the right R-module given by the cokernel of $c$ and the kernel of $z$ is finitely generated and projective.

Equivalently, if $M$ is a right and $L$ a left $R$-module, and $f: M \stackrel{\sim}{\rightarrow} L^{*}$ an isomorphism such that for all R-rings ${ }_{k} T$, the induced homomorphism of $T$ modules $\bar{M} \rightarrow \bar{L}^{*}$ is again an isomorphism, then $M$ and $L$ are (dual) finitely generated projective $R$-modules.

Note that everything we have done in this section goes over (with appropriate minor restatements, e.g., symmetric algebra for tensor-ring) to the analogous constructions in the category of commutative $k$-algebras, though this is definitely false for results of $\S \S 5-11$. Also, in the commutative case we need not assume $k$ a field-see the comment after Lemma 13.2. Since for $R$ a commutative $k$ algebra, a commutative $R$-ring ${ }_{k}$ is just a commutative $R$-algebra, we can drop $k$ entirely in this case.

Turning back to the main idea of this section, the need for $\boldsymbol{P}$ to be finitely generated and projective in Theorem 3.1, we ask similarly whether $P$ in Theorem 3.2 (the construction $R\langle\bar{f}=0\rangle$ where $f: M \rightarrow P$ ) needs to be projective.

The situation is similar to the one we have considered, but more difficult to handle. As above, one can get a weak universal construction for a larger class of modules $P$. Suppose on the other hand that $P$ is an $R$-module for which the theorem holds as stated, for all $M$ and $f$. We find that this condition is not quite strong enough to give the handle we need to characterize $P$. The strengthening we need is shown in condition (ii) of the next result. Even then, we only get a complete characterization when $P$ is finitely generated.

THEOREM 13.6. Let $k$ be a field, $R$ a k-algebra, and $N$ a finitely generated $R$-module, say the image of a map $a: F \rightarrow N$, where $F$ is a free module of finite rank. Then the following conditions are equivalent:

(i) $N$ is projective.

(ii) For every $R$-ring ${ }_{k}, R^{\prime}$, and every homomorphism $f$ of a right $R^{\prime}$. module $M$ into $N \otimes R^{\prime}$, there exists a universal $R^{\prime}$-ring $g_{k} S=R^{\prime}\langle\bar{f}=0\rangle$.

(ii') For every $R$-ring ${ }_{k}, R^{\prime}$, and every element $x \in N \otimes R^{\prime}$, there exists a universal $R^{\prime}$-ring $\left.{ }_{k}, S=R^{\prime} \bar{x}=0\right)$.

(iii) There exists an $R$-ring ${ }_{k}, S=R\langle y \in \bar{F}, \bar{a}(y)=0\rangle$, i.e., an $S$ with universal element of the kernel of $\bar{a}: \bar{F} \rightarrow \bar{N}$.

Proof. (i) $\Rightarrow$ (ii) by Theorem 3.2 .

(ii) $\Leftrightarrow$ (ii'): The forward implication is trivial. On the other hand, assuming (ii') we see that if $X$ is any subset of $N \otimes R^{\prime}$, we can construct $R\langle\bar{X}=\{0\}\rangle$ as the pushout over $R$ of the rings $R \bar{x}=0\rangle(x \in X)$, and we get (ii) by doing this with $X=f(M)$. 
(ii') $\Leftrightarrow$ (iii). To get the forward implication, let $R^{\prime}=R\langle y \in \bar{F}\rangle$, i.e., the extension of $R$ with a universal element of $F Q_{-}$, and apply (ii') with $x=$ $a(y)$. Conversely, assuming (iii) and given $x$ as in (ii'), write $x=\bar{a}(y)$ for some $y \in \bar{F}$; then the ring desired in (ii') is given by the pushout of the diagram:

$$
\begin{aligned}
& R\langle y \in \bar{F}\rangle \longrightarrow R^{\prime} \quad \text { (with } y \in \bar{F} \text { as above) } \\
& R\langle y \in \bar{F}, \bar{a}(y)=0\rangle \quad \text { (given by (iii)). }
\end{aligned}
$$

Assuming the equivalent conditions (ii), (ii'), (iii), we shall now prove (i).

First note that it was by deriving condition (iii) (with $S$ called $U$ ) that in the proof of Theorem 13.3 we proved $N$ finitely related. Hence here again, $N$ will be finitely related.

Now assume (we will justify this later) that $N$ can be defined by one generator and one relation, $N=R / b R(b \in R)$ so that, taking $F=R$, the map $a: F \rightarrow N$ becomes the quotient map $R \rightarrow R / b R$. In particular, the $S$ of condition (iii) (for this possibly new presentation) can be characterized as an $R$-ring ${ }_{k}$ having an element $y \in \bar{b} S$ such that for any $R$-ring ${ }_{k}, T$, and any $u \in \bar{b} T$, there is a unique homomorphism of $R$-rings, $\phi: S \rightarrow T$, taking $y$ to $u$.

Now suppose two homomorphisms of $R$-rings $k_{k}, \alpha, \beta: T \rightrightarrows T^{\prime}$ agree on $u \in \bar{b} T$. Taking $\phi: S \rightarrow T$ as above, and applying the uniqueness condition to $\alpha(u)=\beta(u) \in T^{\prime}$, we conclude that $\alpha \phi=\beta \phi$, i.e., $\phi$ takes $S$ into the difference-kernel subring $T^{\alpha=\beta}={ }_{\operatorname{def}} \operatorname{Ker}(\alpha-\beta) \subseteq T$. Now since $y \in \bar{b} S$, we see $\phi(y)=u \in \bar{b} \phi(S) \subseteq \bar{b} T^{\alpha=\beta}$.

We now consider the canor ${ }^{\circ}$ al map and the zero map of $(R, R)$-bimodules,

$$
R \otimes_{k} R \stackrel{c}{\longrightarrow}(R / R b) \otimes_{k} R
$$

and extend these to maps of $R$-rings s $_{k}: \alpha, \beta: R \oplus(R \otimes R) \rightrightarrows R \oplus(R / R b \otimes R)$. We see that the difference-kernel of these two maps is $T^{\alpha=\beta}=R \oplus(R b \otimes R)$. In particular, this contains the element $u=(0, b \otimes 1) \in \bar{b} T$. So by our preceding observations, $u=(0, b \otimes 1) \in \bar{b}\left(T^{\alpha=\beta}\right)=(b R, b R b \otimes 1)$. Thus, $b \otimes 1 \in$ $b R b \otimes R$, and since $k$ is a field, this says $b \in b R b$. Thus, $b$ is quasi-invertible in $R$, and this makes the module $N=R / b R$ projective! (Explicitly, if $b=$ $b c b$, then $e=b c$ is idempotent and $b R=e R$, so $R / b R=R / e R \cong(1-e) R$.)

To justify our assumption that $N$ had the form $R / b R$, we observe that we can assume $N$ has a presentation with $n$ generators and $n$ relations for some positive integer $n$. Indeed, we can throw in trivial relations if there are too few of these; while if there are fewer generators than relations, enlarge $N$ by adding a free direct summand, which will not affect properties (i) and (ii). Once we have this, we replace $R$ and $N$ by the matrix-ring $\mathfrak{m}_{n}(R)$ and the row- 
vector module $\mathfrak{r}_{n}(N)$. This again will not affect properties (i) and (ii), and will reduce the $n$ generators and $n$ relations defining $N$ to one generator and one relation, as desired. (This reduction was not essential, but just served to make the main calculation more transparent. If we want to prove the same result in the category of commutative $k$-algebras, we will probably have to do without it.)

\section{REFERENCES}

1. S. A. Amitsur, Embeddings in matrix rings, Pacific J. Math. 36 (1971), 21-29. MR 43 \#2017.

2. Hyman Bass, Algebraic K-theory, Benjamin, New York, 1968. MR 40 \#2736.

3. George $\mathrm{M}$. Bergman, Hereditary commutative rings and centres of hereditary rings, Proc. London Math. Soc. (3) 23 (1971), 214-236. MR 46 \#9022.

4. - Infinite multiplication of ideals in $\aleph_{0}$-hereditary rings, J. Algebra 34 (1973), 56-70. MR 46 \#9085.

5. - Rational relations and rational identities in division rings I, J. Algebra (to appear). (Note: [9] is a prerequisite to [5].)

6. - Some examples in p.i. ring theory, Israel J. Math. (to appear).

7. Modules over coproducts of rings, Trans. Amer. Math. Soc. 200 (1974), 1-32.

8. - The diamond lemma for ring theory (to appear).

9. George M. Bergman and Lance W. Small, P.i. degrees and prime ideals (to appear).

10. A. J. Bowtell, On a question of Mat'cev, J. Algebra 7 (1967), 126-139. MR 37 \#6310.

11. W. Edwin Clark and George M. Bergman, The automorphism class group of the category of rings, J. Algebra 24 (1973), 80-99.

12. P. M. Cohn, Universal algebra, Harper and Row, New York, 1965. MR 31 \#224.

13. - Some remarks on the invariant basis property, Topology 5 (1966), 215-

228. MR 33 \#5676.

14. - The embedding of firs in skew fields, Proc. London Math. Soc. (3) 23

(1971), 193-213. MR 45 \#6866.

15. - Dependence in rings. II. The dependence number, Trans. Amer. Math. Soc. 135 (1969), 267-279. MR 43 \#4848.

16. - Free rings and their relations, Academic Press, New York, 1971.

17. - Rings of fractions, Lecture Notes, University of Alberta, Edmonton, Alberta, Canada, 1972.

18. - Localization in semifirs (to appear).

19. S. Eilenberg, T. Nakayama and H. Nagao, On the dimension of modules and algebras. IV. Dimension of residue rings of hereditary rings, Nagoya Math. J. 10 (1956), 8795. MR 18, 9.

20. K. L. Fields, On the global dimension of residue rings, Pacific J. Math. 32 (1970), 345-349. MR 42 \#6049.

21. Peter Freyd, Algebra valued functors in general and tensor products in particular, Colloq. Math. 14 (1966), 89-106. MR 33 \#4116.

22. J. R. Isbell, Epimorphisms and dominions. IV, J. London Math. Soc. (2) 1 (1969), 265-273.

23. A. A. Klein, Rings nonembeddable in fields with multiplicative semigroups embeddable in groups, J. Algebra 7 (1967), 100-125. MR 37 \#6309. 
24. A. A. Klein, A remark concerning embeddability of rings in fields, J. Algebra 21 (1972), 271-274. MR 45 \#8670.

25. W. G. Leavitt, Modules without invariant basis number, Proc. Amer. Math. Soc. 8 (1957), 322-328. MR 18, 789.

26. Saunders Mac Lane, Categories for the working mathematician, Graduate Texts in Math., no. 5, Springer-Verlag, New York, 1971.

27. A. I. Mal'cev, Über die Einbettung von assoziativen Systemen in Gruppen. I, Mat. Sb. 6 (48) (1939), 331-336. (Russian) MR 2, 7.

28. B. Mitchell, Rings with several objects, Advances in Math. 8 (1972), 1-161.

29. L. Silver, Noncommutative localizations and applications, J. Algebra 7 (1967), 44-76. MR 36 \#205.

30. L. A. Skornjakov, On Cohn rings, Algebra i Logika Sem. 4 (1965), no. 3, 5-30. (Russian) MR 33 \#1326.

31. Lance W. Small, Hereditary rings, Proc. Nat. Acad. Sci. U.S.A. 55 (1966), $25-27$. MR 32 \#4178.

32. George M. Bergman, Epimorphisms of Lie algebras (to appear).

33. F. Waldhausen, "Whitehead groups of generalized free products," in Algebraic K-theory. II: "Classical" algebraic K-theory and connections with arithmetic, H. Bass (editor), Lecture Notes in Math., no. 342, Springer-Verlag, Berlin, 1973, pp. 155-182.

DEPARTMENT OF MATHEMATICS, UNIVERSITY OF CALIFORNIA, BERKELEY, CALIFORNIA 94720 